\title{
$\begin{array}{ll}\text { Research Square } & \text { Preprints are preliminary reports that have not undergone peer review. } \\ \text { They should not be considered conclusive, used to inform clinical practice, }\end{array}$ or referenced by the media as validated information. \\ Numerical and Experimental Investigations on a Friction Ring Damper for a Flywheel
}

\section{Xiaodong He}

Shanghai Jiaotong University: Shanghai Jiao Tong University

\section{Zhiwei Zheng}

Shanghai Jiaotong University: Shanghai Jiao Tong University

\section{Xiuchang Huang ( $\nabla$ xchhuang@sjtu.edu.cn)}

Shanghai Jiaotong University: Shanghai Jiao Tong University https://orcid.org/0000-0002-0013-3335

\section{Sen Wang}

Shanghai Aerospace Control Technology Reasearch Institute

\section{Xinsheng Wei}

Shanghai Aerospace Control Technology Research Institute

\section{Yong Wang}

Shanghai Aerospace Control Technology Research Institute Hongxing Hua

Shanghai Jiaotong University: Shanghai Jiao Tong University

\section{Research Article}

Keywords: flywheel, friction ring damper, nonlinear dynamics, HBM, spectral testing

Posted Date: December 6th, 2021

DOI: https://doi.org/10.21203/rs.3.rs-1123961/v1

License: (c) (1) This work is licensed under a Creative Commons Attribution 4.0 International License.

Read Full License 


\title{
Numerical and Experimental Investigations on a Friction Ring Damper for a Flywheel
}

Xiaodong $\mathrm{He}^{1}$, Zhiwei Zheng ${ }^{1}$, Xiuchang Huang ${ }^{1 *}$, Sen Wang ${ }^{2}$, Xinsheng $\mathrm{Wei}^{2}$, Yong Wang ${ }^{2}$ and Hongxing Hua ${ }^{1}$

\footnotetext{
${ }^{1}$ Fundamental Science on Vibration, Shock and Noise Laboratory, State Key Laboratory of Mechanical System and Vibration, Shanghai Jiaotong University, Shanghai, China, 200240

${ }^{2}$ Shanghai Aerospace Control Technology Research Institute, Shanghai, China, 201109 ("Corresponding author: Xiuchang Huang. E-mail address: xchhuang@ @jtu.edu.cn)
}

\begin{abstract}
A damping strategy using a friction ring damper for an industrial flywheel was numerically and experimentally investigated. The friction ring damper, located on the arms of the flywheel, was experimentally found to effectively reduce the vibration amplitude of the flywheel. The vibration energy is dissipated when relative motions occur at the friction contact interfaces. Nonlinear dynamic analysis based on a lumped-parameter model of a flywheel equipped with a friction ring damper was conducted. A dimensionless parameter, $\kappa$, defined as the ratio of the critical friction force to the amplitude of harmonic force, was used to evaluate the damping performance. For several values of $\kappa$, steady-state responses under harmonic excitation and nonlinear modes were obtained using the harmonic balance method (HBM) combined with the alternating frequency-time domain method (AFT). The forced response analysis proved the existence of an optimal value of $\kappa$, which can minimize the vibration amplitude of the flywheel. The nonlinear modal analysis showed that all the damping ratio-frequency curves are completely coincident even for different $\kappa$, and the frequency corresponding to the maximum damping ratio is equal to the frequency at the intersection of the forced response curves under the fully
\end{abstract}


slip and the fully stick states of the friction contact interface. By analyzing the behaviors of the friction contact interface, it is shown that the friction contact interface provides damping in the combined stickslip state. The forced response under random excitation was calculated using the Runge-Kutta method and the friction interface behaviors were analyzed. Finally, spectral testing was conducted to verify the numerical results.

Keywords: flywheel, friction ring damper, nonlinear dynamics, HBM, spectral testing

\section{Introduction}

This study focused on the vibration suppression of flywheels. Flywheels are widely used to control the attitude and maintain the stability of satellites. In the launch phase of the rocket, the flywheel is excited to a violent resonance under severe random vibrations. According to estimations based on the resonance intensity of the flywheel, the force acting on the bearing caused by the vibration amplification of the wheel body greatly exceeds the static load capacity of the bearing, putting the bearing at risk of damage. Meanwhile, the inherent damping in the flywheel is very low, and there is nearly no source of

energy dissipation. Therefore, the flywheel's ability to withstand these vibrations mainly depends on the external inputs of damping; therefore, it is necessary to develop new methods and devices to damp the vibrations of the flywheel.

In previous studies, the use of a flexible dynamic vibration absorber (DVA) was proposed to suppress the high-frequency micro vibrations of the flywheel [1-3], in this method, rubber pads were used to increase the stiffness and damping of the DVA. In this study, friction pads made of epoxy phenolic glass cloth were used to replace the rubber pads in the damping ring, the elastic modulus of the friction pad is $1 \mathrm{GPa}$. Thus, the original flexible DVA became a dry friction damper mounted on the arms of the flywheel, in which contact is permanently maintained by the preload of the bolts. Friction thus occurs at the friction contact interfaces when relative motions occur between the friction pads and arms of the flywheel to dissipate the vibration energy. The friction at the contact interface is the most widely used 
source of external damping in bladed disk assemblies [4] and blisks (integrally bladed disks) [5], which include shroud contact in blades, platform dampers, and friction rings. Our work drew upon numerous methods of design and analysis of these devices reported in the literature. The HBM [6] is the most widely used method for the nonlinear steady-state response prediction of bladed disk assemblies and blisks with friction dampers [7-11], which is a computationally efficient and accurate method to perform dynamic analyses quantitatively. The nonlinear modal analysis of the bladed disks with friction contact interfaces $[12,13]$ is based on the concept of complex nonlinear modes [14] and the frequency domain formulation of the dynamical system's equations of motion [15]. The AFT method [16] is used for calculating the implicit nonlinear friction forces. Recently, the HBM combined with the AFT to calculate the friction force in the time domain and the arc-length continuation technique to improve the convergence of the method [17] were used when performing nonlinear forced response [18, 19] and nonlinear modal analysis [20].

Although the bladed disk assemblies and blisks with friction dampers have been extensively studied, little numerical or experimental research has been performed on the friction ring damper for flywheels. Motivated by this gap in research, in this study, an equivalent lumped-parameter model of the flywheel with friction contact interfaces was established to study the dynamic behavior of the system. The vibration suppression performance of the damper is discussed based on nonlinear forced response and nonlinear modal analysis. In addition, spectral testing was conducted to verify the numerical results.

The remainder of this paper is organized into five sections: the lumped-parameter model of the friction ring damper-flywheel-bearing system is described and the governing equation of the system presented in Section 2. The numerical analysis (nonlinear modal and nonlinear forced response analysis based on HBM combined with AFT) is described in Section 3. The numerical results and related discussions are presented in Section 4. The spectral testing on the flywheel conducted with and without the friction ring damper to verify the numerical results is discussed in Section 5. The conclusions are presented in Section 6. 


\section{Dynamic modelling}

As shown in Figure 1, the characteristic mode of the flywheel obtained from the finite element model is an umbrella-shaped flapping mode, which is dominated by the axial movement of the wheel body and the bearing resting on the disc spring [1]. The flapping mode is the main contributor to the axial force acting on the bearing owing to a unit force applied at the rim of the flywheel. The aim of this study is to reduce the vibration amplitude of the flywheel and mitigate the axial force acting on the bearing.
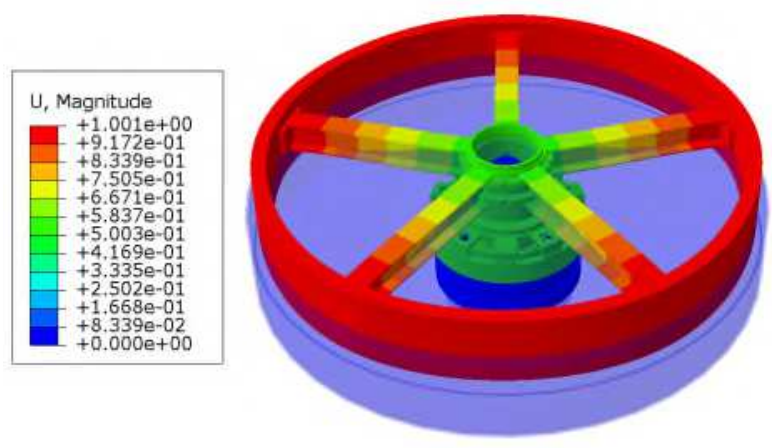

Fig. 1 Umbrella-shaped flapping mode of the flywheel.

As shown in Figure 2(a), the friction ring damper is attached to the arms of the flywheel. When relative motions occur at the contact interfaces between the friction pads and arms, the vibration energy of the flywheel is dissipated by friction damping. An equivalent 11-degree of freedom (DOF) lumpedparameter model of the flywheel-bearing system equipped with the friction ring damper is developed as shown in Figure 2(b). Where $m_{\mathrm{f}, i}=m_{\mathrm{f}}(i=1,2, \ldots, 5)$ and $k_{\mathrm{f}}$ denote the masses and stiffness of the flywheel, respectively; $k_{\mathrm{f} 0}$ denotes the connection stiffness; $m_{\mathrm{b}}$ and $k_{\mathrm{b}}$ denote the mass and stiffness of the bearing, respectively; $m_{\mathrm{r}, i}=m_{\mathrm{r}}(i=1,2, \ldots, 5)$ and $k_{\mathrm{r} 0}$ denote the masses and the connection stiffness of the damping ring, respectively; $c_{\mathrm{r}}$ denotes the viscous damping; and $k_{\mathrm{g}}$ denotes the connection stiffness to the ground. Five external forces, $F=0.2 \cos (\omega t)$, were uniformly applied at the five flywheel masses. The numerical values of the parameters of the model are listed in Table 1. 


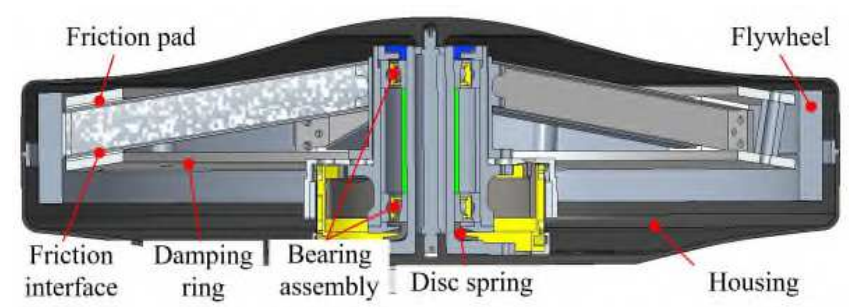

(a)

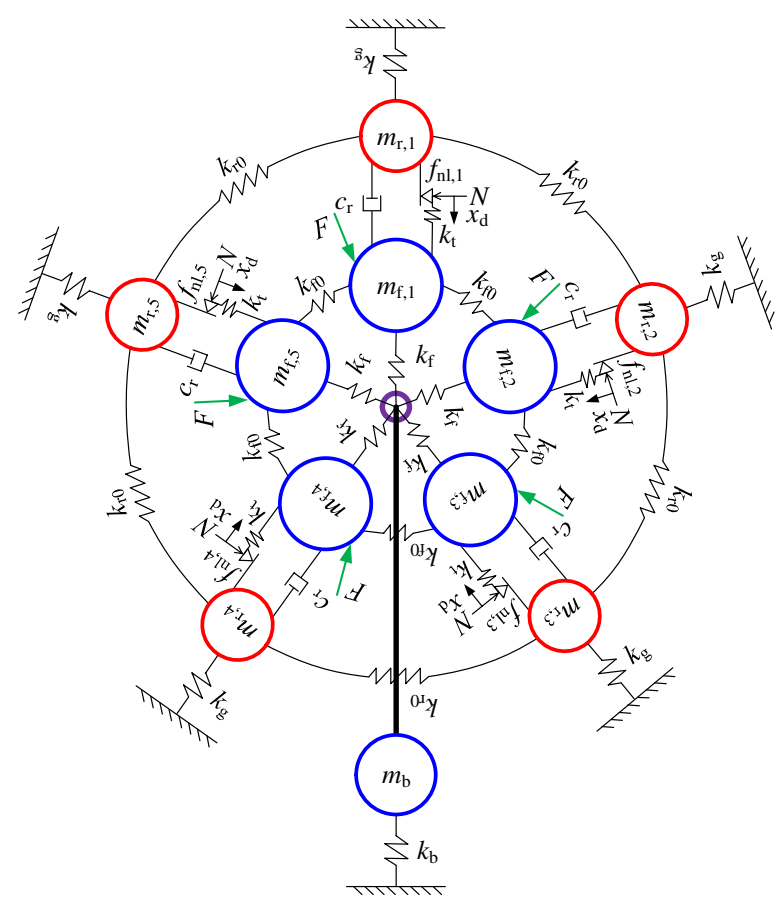

(b)

Fig. 2 Flywheel equipped with the friction ring damper (a) and the 11-DOF lumped-parameter model (b).

Table 1 Parameter values of the lumped-parameter model.

\begin{tabular}{|c|c|c|c|c|}
\hline$m_{\mathrm{f}}$ & $k_{\mathrm{f}}$ & $k_{\mathrm{f} 0}$ & $m_{\mathrm{b}}$ & $k_{\mathrm{b}}$ \\
\hline $0.9105 \mathrm{~kg}$ & $2.824 \times 10^{6} \mathrm{~N} / \mathrm{m}$ & $1 \times 10^{7} \mathrm{~N} / \mathrm{m}$ & $1.0710 \mathrm{~kg}$ & $1.6150 \times 10^{7} \mathrm{~N} / \mathrm{m}$ \\
\hline$m_{\mathrm{r}}$ & $k_{\mathrm{g}}$ & $k_{\mathrm{r} 0}$ & $c_{\mathrm{r}}$ & - \\
\hline $0.0990 \mathrm{~kg}$ & $1 \times 10^{10} \mathrm{~N} / \mathrm{m}$ & $1 \times 10^{6} \mathrm{~N} / \mathrm{m}$ & 0 & - \\
\hline
\end{tabular}

The friction contact interfaces between the flywheel and the damping ring were modelled based on the Coulomb friction model, the Coulomb friction force is expressed as $[21,18]$

$$
f_{\mathrm{nl}, i}=\left\{\begin{array}{lc}
k_{\mathrm{t}}\left(z_{i}-x_{\mathrm{d}, i}\right) & \text { stick } \\
f_{\mathrm{c}} \operatorname{sgn}\left(\&_{\mathrm{q}, i}\right) & \text { slip }
\end{array},\right.
$$

where $k_{\mathrm{t}}$ is the tangential stiffness of the friction contact interface, $z_{i}=x_{\mathrm{f}, i}-x_{\mathrm{r}, i}$ is the relative displacement between the flywheel and the damping ring, $x_{\mathrm{d}, i}$ is the displacement of the friction contact point, $f_{\mathrm{c}}=\mu_{\mathrm{f}} N$ is the critical friction force where $\mu_{\mathrm{f}}$ is the coefficient of friction, and $N$ is the normal load. 
The governing equation of the equivalent 11-DOF lumped-parameter model of the system is

$$
\mathbf{M} \mathbf{C} \& \mathbf{K} \mathbf{x}+\mathbf{F}_{\mathrm{nl}}(\mathbf{x}, \mathbf{x})=\mathbf{F}_{\mathrm{ext}},
$$

where $\mathbf{M}, \mathbf{C}$, and $\mathbf{K}$ are the mass matrix, damping matrix, and stiffness matrix, respectively; $\mathbf{x}$ \& , and are the displacement vector, velocity vector, and acceleration vector, respectively; and $\mathbf{F}_{\mathrm{nl}}$ and $\mathbf{F}_{\mathrm{ext}}$ are the nonlinear friction force vector and external force vector, respectively.

The matrices and vectors in Eq. (2) are given by

$$
\begin{aligned}
& \mathbf{M}=\left[\begin{array}{ccc}
\mathbf{M}_{\mathrm{r}} & & \\
& \mathbf{M}_{\mathrm{f}} & \\
& & m_{\mathrm{b}}
\end{array}\right], \quad \mathbf{M}_{\mathrm{r}}=\operatorname{diag}\left\{m_{\mathrm{r}}\right\}_{5 \times 5}, \quad \mathbf{M}_{\mathrm{f}}=\operatorname{diag}\left\{m_{\mathrm{f}}+m_{\mathrm{r}}\right\}_{5 \times 5}, \\
& \mathbf{C}=\left[\begin{array}{ccc}
\mathbf{C}_{\mathrm{r}} & -\mathbf{C}_{\mathrm{r}} & \\
-\mathbf{C}_{\mathrm{r}} & \mathbf{C}_{\mathrm{r}} & \\
& & 0
\end{array}\right], \quad \mathbf{C}_{\mathrm{r}}=\operatorname{diag}\left\{c_{\mathrm{r}}\right\}_{5 \times 5} \\
& \mathbf{K}=\left[\begin{array}{ccc}
\mathbf{K}_{\mathrm{rr}} & \mathbf{0}_{5 \times 5} & \mathbf{0}_{5 \times 1} \\
\mathbf{0}_{5 \times 5} & \mathbf{K}_{\mathrm{ff}} & \left\{-k_{\mathrm{f}}\right\}_{5 \times 1} \\
\mathbf{0}_{1 \times 5} & \left\{-k_{\mathrm{f}}\right\}_{1 \times 5} & 5 k_{\mathrm{f}}+k_{\mathrm{b}}
\end{array}\right] \\
& \mathbf{K}_{\mathrm{rr}}=\left[\begin{array}{ccccc}
2 k_{\mathrm{r} 0}+k_{\mathrm{g}} & -k_{\mathrm{r} 0} & 0 & 0 & -k_{\mathrm{r} 0} \\
-k_{\mathrm{r} 0} & \cdot & \cdot & 0 & 0 \\
0 & \cdot & . & . & 0 \\
0 & 0 & . & . & -k_{\mathrm{r} 0} \\
-k_{\mathrm{r} 0} & 0 & 0 & -k_{\mathrm{r} 0} & 2 k_{\mathrm{r} 0}
\end{array}\right], \mathbf{K}_{\mathrm{ff}}=\left[\begin{array}{ccccc}
k_{\mathrm{f}}+2 k_{\mathrm{f} 0} & -k_{\mathrm{f} 0} & 0 & 0 & -k_{\mathrm{f} 0} \\
-k_{\mathrm{f} 0} & \cdot & \cdot & 0 & 0 \\
0 & \cdot & . & \cdot & 0 \\
0 & 0 & . & . & -k_{\mathrm{f} 0} \\
-k_{\mathrm{f} 0} & 0 & 0 & -k_{\mathrm{f} 0} & k_{\mathrm{f}}+2 k_{\mathrm{f} 0}
\end{array}\right],
\end{aligned}
$$

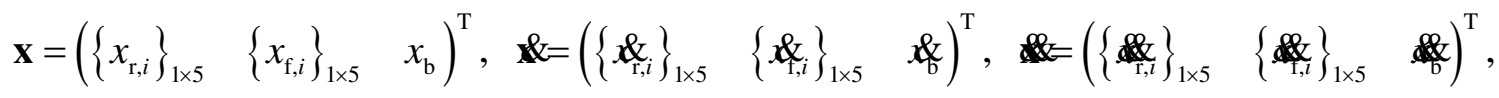

$$
\begin{aligned}
& \mathbf{F}_{\mathrm{nl}}=\left(\{1\}_{1 \times 5} \quad\{-1\}_{1 \times 5} \quad 0\right)^{\mathrm{T}} \cdot f_{\mathrm{nl}}, \quad \mathbf{F}_{\mathrm{ext}}=\left(\begin{array}{ll}
\{0\}_{1 \times 5} \quad\{1\}_{1 \times 5} & 0
\end{array}\right)^{\mathrm{T}} \cdot F .
\end{aligned}
$$

\section{Numerical analysis}

In this section, the nonlinear mode and nonlinear forced response of the flywheel equipped with the friction ring damper are derived using HBM [11] combined with AFT [16]. Without loss of generality, 
the HBM-AFT method is presented for a general multi-DOF dynamic system with a general nonlinear force.

\subsection{Nonlinear modal analysis}

Nonlinear modal analysis is performed based on the HBM for $\mathbf{F}_{\mathrm{ext}}=\mathbf{0}$ in Eq. (2) by assuming that

$$
\begin{aligned}
& \mathbf{x}(t)=\mathbf{Q}^{0}+\sum_{k=1}^{N}\left(\mathbf{Q}^{k, c} \cos k \omega t+\mathbf{Q}^{k, s} \sin k \omega t\right), \\
& \mathbf{F}_{\mathrm{nl}}(t)=\mathbf{F}_{\mathrm{nl}}^{0}+\sum_{k=1}^{N}\left(\mathbf{F}_{\mathrm{nl}}^{k, c} \cos k \omega t+\mathbf{F}_{\mathrm{nl}}^{k, s} \sin k \omega t\right),
\end{aligned}
$$

where $N$ is the retained number of harmonics.

The vectors of the Fourier coefficients in Eqs. (9) and (10) are

$$
\begin{gathered}
\mathbf{Q}=\left\{\begin{array}{llllll}
\mathbf{Q}^{0} & \mathbf{Q}^{1, c} & \mathbf{Q}^{1, s} & \ldots & \mathbf{Q}^{N, c} & \mathbf{Q}^{N, s}
\end{array}\right\}^{\mathrm{T}}, \\
\mathbf{F}_{\mathrm{nl}}=\left\{\begin{array}{llllll}
\mathbf{F}_{\mathrm{nl}}^{0} & \mathbf{F}_{\mathrm{nl}}^{1, c} & \mathbf{F}_{\mathrm{nl}}^{1, s} & \ldots & \mathbf{F}_{\mathrm{nl}}^{N, c} & \mathbf{F}_{\mathrm{nl}}^{N, s}
\end{array}\right\}^{\mathrm{T}},
\end{gathered}
$$

where

$$
\mathbf{F}_{\mathrm{nl}}^{0}=\frac{1}{T} \int_{-T / 2}^{T / 2} \mathbf{F}_{\mathrm{nl}}\left(\mathbf{x}, \mathbf{\mathrm { d } t}, \quad \mathbf{F}_{\mathrm{nl}}^{k, c}=\frac{2}{T} \int_{-T / 2}^{T / 2} \mathbf{F}_{\mathrm{nl}}\left(\mathbf{x}, \cos k \omega t \mathrm{~d} t, \quad \mathbf{F}_{\mathrm{nl}}^{k, s}=\frac{2}{T} \int_{-T / 2}^{T / 2} \mathbf{F}_{\mathrm{nl}}(\mathbf{x}, \boldsymbol{s i n} k \omega t \mathrm{~d} t\right.\right.
$$

Then Eq. (2) can be expressed as

$$
\mathbf{M} \otimes \mathbf{I}_{2 N+1} \mathbf{B}^{2}+\mathbf{C} \otimes \mathbf{I}_{2 N+1} \mathbf{B}+\mathbf{K} \otimes \mathbf{I}_{2 N+1}+\mathbf{F}_{\mathrm{nl}}(\mathbf{x}, \boldsymbol{\otimes})=\mathbf{0},
$$

where $\otimes$ is the Kronecker product, and

$$
\mathbf{B}=\operatorname{diag}\left(\begin{array}{lllll}
\mathbf{0} & \boldsymbol{\Lambda}_{1} & \boldsymbol{\Lambda}_{2} & \ldots & \boldsymbol{\Lambda}_{2 N+1}
\end{array}\right), \quad \boldsymbol{\Lambda}_{k}=k\left[\begin{array}{cc}
0 & \omega \\
-\omega & 0
\end{array}\right]
$$

The AFT procedure is employed to obtain the frequency-domain components of the nonlinear friction force

$$
\mathbf{Q} \rightarrow \mathbf{x}(\omega) \stackrel{\mathrm{IFFT}}{\longrightarrow} \mathbf{x}(t) \stackrel{\mathbf{B}}{\longrightarrow}(t) \rightarrow \mathbf{F}_{\mathrm{nl}}\left(\mathbf{x}, \stackrel{\mathrm{FFT}}{\longrightarrow} \mathbf{F}_{\mathrm{nl}}(\mathbf{Q}, \omega)\right.
$$

This gives the following set of the nonlinear algebraic equations 


$$
\mathbf{H}(\mathbf{Q}, \omega)=\mathbf{M} \otimes \mathbf{I}_{2 N+1} \mathbf{B}^{2}+\mathbf{C} \otimes \mathbf{I}_{2 N+1} \mathbf{B}+\mathbf{K} \otimes \mathbf{I}_{2 N+1}-\mathbf{F}_{\mathrm{nl}}(\mathbf{Q}, \omega)=\mathbf{0} .
$$

Then, the Newton-Raphson method is employed to solve Eq. (17) and obtain the nonlinear modes.

\subsection{Forced response analysis}

The forced responses are also obtained using the Newton-Raphson method to solve the nonlinear algebraic equation

$$
\mathbf{H}(\mathbf{Q}, \omega)=\mathbf{M} \otimes \mathbf{I}_{2 N+1} \mathbf{B}^{2}+\mathbf{C} \otimes \mathbf{I}_{2 N+1} \mathbf{B}+\mathbf{K} \otimes \mathbf{I}_{2 N+1}-\mathbf{F}_{\mathrm{nl}}(\mathbf{Q}, \omega)-\mathbf{F}_{\mathrm{ext}}(\mathbf{Q}, \omega)=\mathbf{0}
$$

where the external force vector $\mathbf{F}_{\text {ext }}(\mathbf{Q}, \omega)$ can be expressed in terms of a truncated Fourier series

$$
\mathbf{F}_{\mathrm{ext}}(t)=\mathbf{F}_{\mathrm{ext}}^{0}+\sum_{k=1}^{N}\left(\mathbf{F}_{\mathrm{ext}}^{k, c} \cos k \omega t+\mathbf{F}_{\mathrm{ext}}^{k, s} \sin k \omega\right)
$$

The vector of the Fourier coefficients of the external force is

$$
\mathbf{F}_{\mathrm{ext}}=\left\{\begin{array}{llllll}
\mathbf{F}_{\mathrm{ext}}^{0} & \mathbf{F}_{\mathrm{ext}}^{1, c} & \mathbf{F}_{\mathrm{ext}}^{1, s} & \ldots & \mathbf{F}_{\mathrm{ext}}^{N, c} & \mathbf{F}_{\mathrm{ext}}^{N, s}
\end{array}\right\}^{\mathrm{T}}
$$

This was solved using an arc-length continuation-based prediction-correction algorithm [17-19].

\section{Results and discussions}

\subsection{Nonlinear forced responses}

When the friction contact interface is in the fully stick state $(N=\infty)$, the only connector between the flywheel and damping ring is the tangential stiffness spring $k_{\mathrm{t}}$. The nonlinear forced responses of the flywheel under harmonic excitation for several values of $k_{\mathrm{t}}$ were obtained and plotted, as shown in Figure 3. It can be seen that when the friction contact interface is in the fully stick state, the frequency increases monotonically as $k_{\mathrm{t}}$ increases. A modal test was performed on the system to determine the value of $k_{\mathrm{t}}$, and the experimental results showed that the frequency of the flapping mode was $200 \mathrm{~Hz}$. Thus, $k_{\mathrm{t}}$ was taken as $2 \times 10^{4} \mathrm{~N} / \mathrm{m}$ in the lumped-parameter model, and the frequency obtained from simulation is 200.7 Hz when the friction contact interface is in the fully stick state $(N=\infty)$ and $199.4 \mathrm{~Hz}$ when it is in the fully slip state $\left(k_{\mathrm{t}}=0\right)$. 


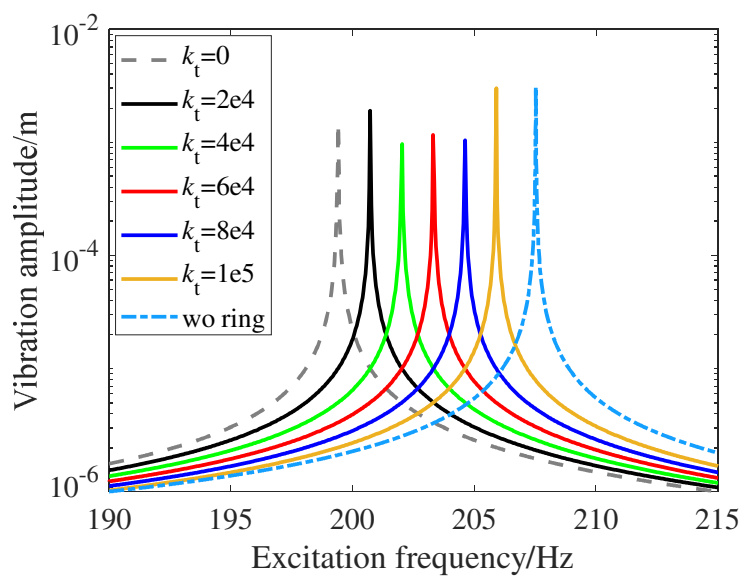

Fig. 3 Effect of $k_{\mathrm{t}}$ on the frequency of the flywheel.

We define the ratio of the critical friction force $\mu_{\mathrm{f}} N$ to the amplitude of harmonic force $F$ as a dimensionless parameter $\kappa=\mu_{\mathrm{f}} N / F$. The nonlinear forced responses of the flywheel were obtained with several values of $\kappa$, as shown in Figure 4 (Figure 4(b) is an enlarged view of Figure 4(a)). It can be seen that as $\kappa$ gradually increases from 0 to $\infty$, the vibration amplitude of the flywheel first decreases and then increases, and the friction contact interface undergoes a transition from the fully slip $(\kappa=0, N=0)$ to the fully stick state $(\kappa=\infty, N=\infty)$. The qualitative results are similar to those for bladed disks with underplatform dampers or friction rings [6]. When $\kappa$ is in the range of $4-15$, the friction ring damper greatly and effectively reduces the vibration amplitude of the flywheel. When $\kappa$ is in the range of 5-8, an optimal value of $\kappa$ exists that can minimize the vibration amplitude.

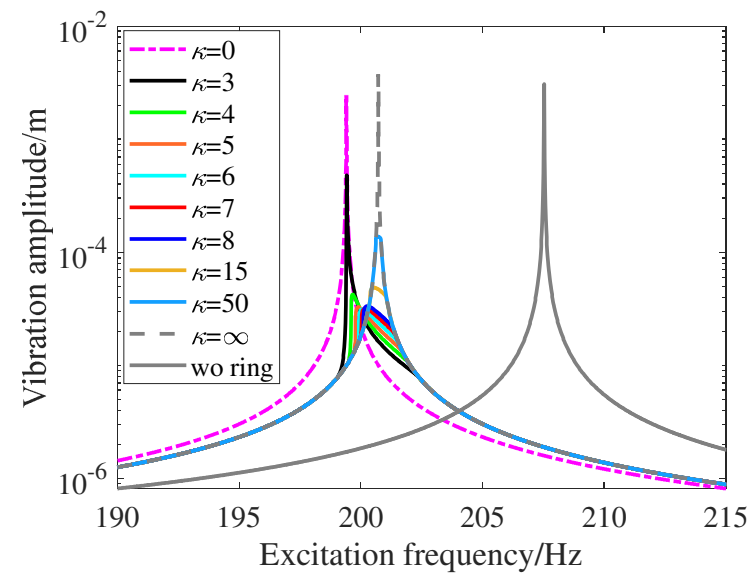

(a)

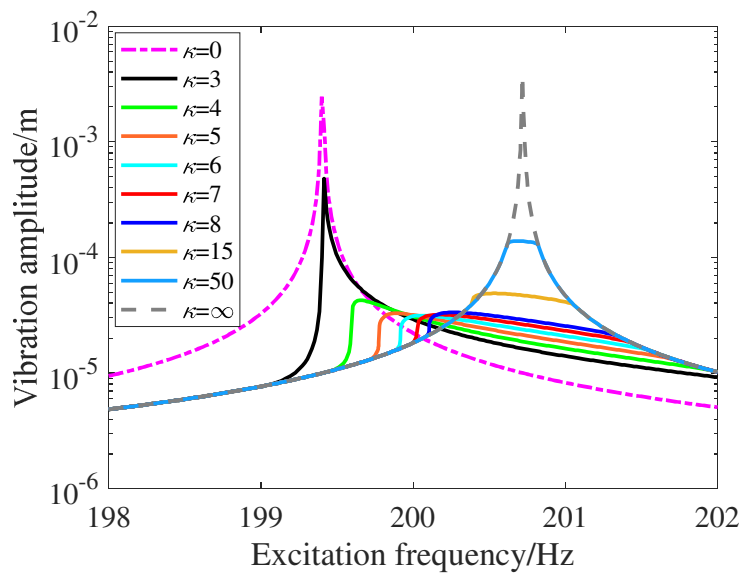

(b)

Fig. 4 Effect of $\kappa$ on the vibration amplitude of the flywheel. 


\subsection{Nonlinear modes and behaviors of the friction interface}

Figures 5(a) and (b) show the frequency-modal amplitude and damping ratio-modal amplitude diagrams, respectively, for several values of $\kappa$. As $\kappa$ increases, the nonlinear modal curves translate in the direction in which the modal amplitude becomes larger. The damping ratio-frequency diagrams at the modal amplitude shown in Figures 5(a) and (b) are presented in Figure 5(c). It can be seen from Figure 5(d) that all the damping ratio-frequency curves are fully coincident even for different $\kappa$, and the frequency at the maximum damping ratio is equal to the intersection frequency of the forced response curves for $\kappa=0$ (fully slip state) and $\kappa=\infty$ (fully stick state).

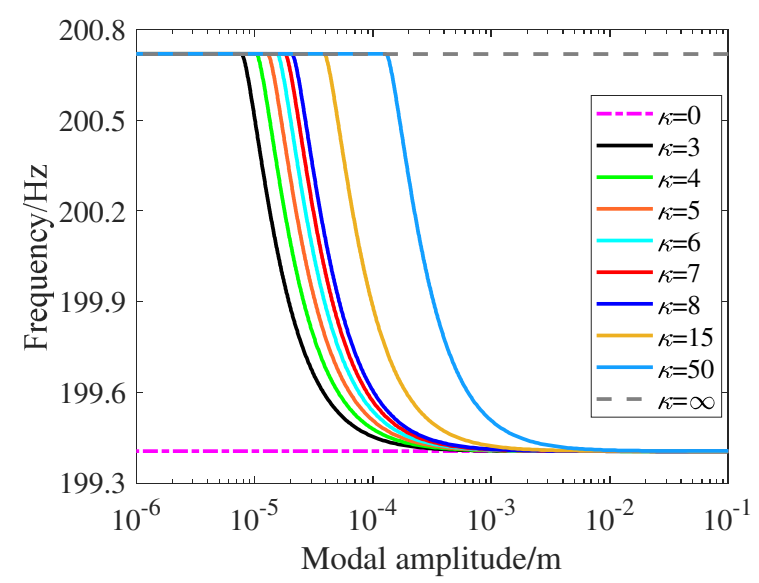

(a)

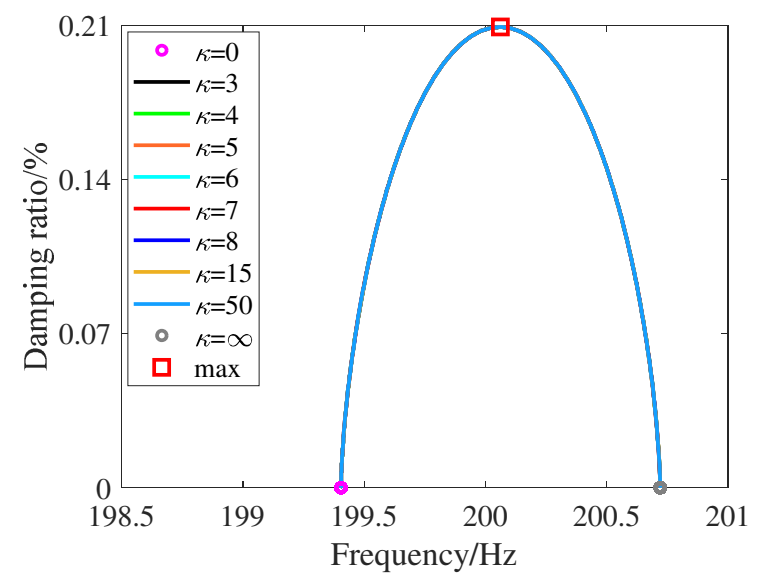

(c)

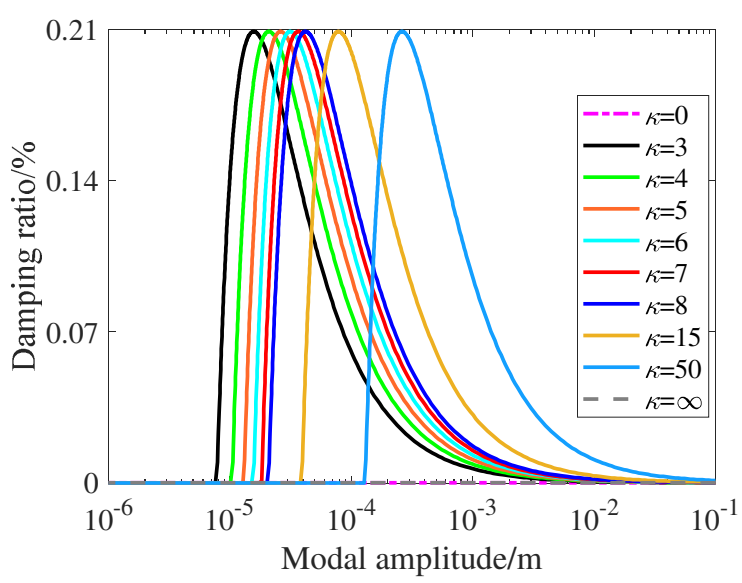

(b)

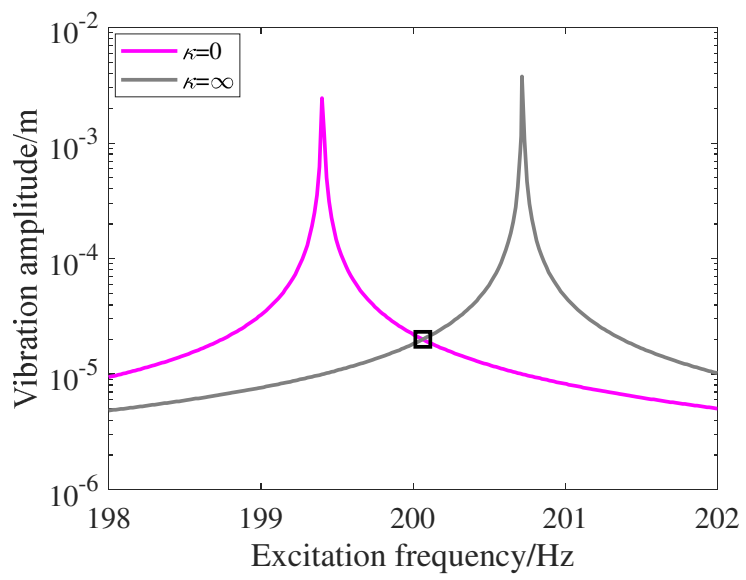

(d)

Fig. 5 Frequency-modal amplitude diagram (a), damping ratio-modal amplitude diagram (b), damping ratiofrequency diagram (c), and intersection of the forced response curves for $\kappa=0$ and $\kappa=\infty(\mathrm{d})$. 
The optimal value of $\kappa$ was found to be 6.07 by employing a genetic algorithm [3]. The nonlinear forced responses of the flywheel at $\kappa=0, \kappa=\infty$, and $\kappa=$ opt (the optimal value of $\kappa$ ) are shown in Figure 6(a), where $f_{\text {slip }}$ and $f_{\text {stick }}$ are the frequencies when the friction contact interface is in the fully slip and fully stick state, respectively. It can be seen that the vibration amplitude of the flywheel is the smallest at $\kappa=$ opt, and the frequency at the maximum vibration amplitude is equal to the frequency at the intersection of the response curves for $\kappa=0$ and $\kappa=\infty$.

The nonlinear modes at $\kappa=$ opt are showed in Figure 6(b), where some feature points on the nonlinear modal curves are marked out using small squares (points a-f). As shown in Figure 6(b), when the vibration amplitude of the flywheel is less than the modal amplitude at point a, the friction contact interface is in the fully stick state. Point a is a state transition point, where the state of the friction contact interface begins to change from the fully stick to the combined stick-slip state as the modal amplitude increases. At points b and c, the friction contact interface is in the combined stick-slip state, i.e., the stick and slip states coexist. At points $d$ and e, the combined stick-slip state gradually approaches the fully slip state. When the vibration amplitude of the flywheel exceeds the modal amplitude at point $\mathrm{f}$, the friction contact interface is in a fully slip state. In the state transition of the friction contact interface from the fully stick to the fully slip state, the frequency decreases monotonously as the modal amplitude increases from point a to f. Correspondingly, the damping ratio increases rapidly from 0 (the fully stick state at point a) to the maximum value (the combined stick-slip state at point c) and then slowly decreases to 0 (the fully slip state at point $\mathrm{f}$ ).

The nonlinear forced response curve and nonlinear modal curve at $\kappa=$ opt (Figures 6(a) and (b)) are plotted on the same graph in Figure 6(c). The vibration amplitudes of the flywheel at point 1 and 5 on the nonlinear forced response curve are the same as the modal amplitude at point a on the nonlinear modal curve. As discussed earlier, the friction contact interface is in the fully stick state at points 1 and 5. Point 3 is the peak of the nonlinear forced response curve, at which point the vibration amplitude is the same as the modal amplitude at point $\mathrm{c}$ on the nonlinear modal curve. The frequency range between 
$f_{\mathrm{s}}$ at point 1 and $f_{\mathrm{e}}$ at point 5 is the non-coinciding section for the forced response curves at $\kappa=$ opt and $\kappa=\infty$. The vibration amplitudes of this section lie between the modal amplitudes at points a and $\mathrm{c}$. Therefore, in the non-coinciding section (Figure 6(b)), the friction contact interface is in the combined stick-slip state, and the friction contact interface is able to provide damping.

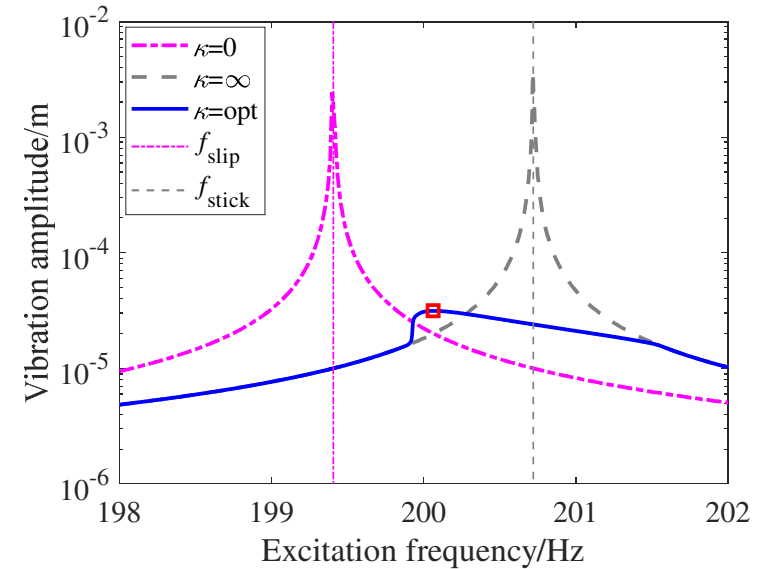

(a)
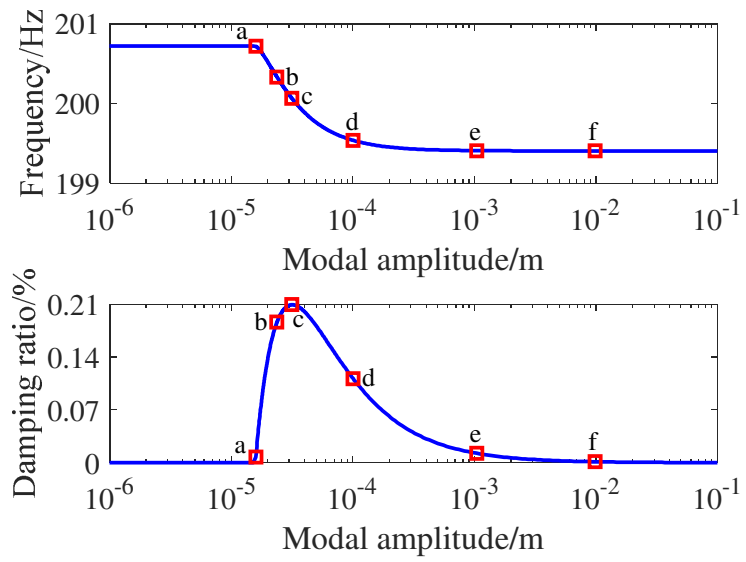

(b)

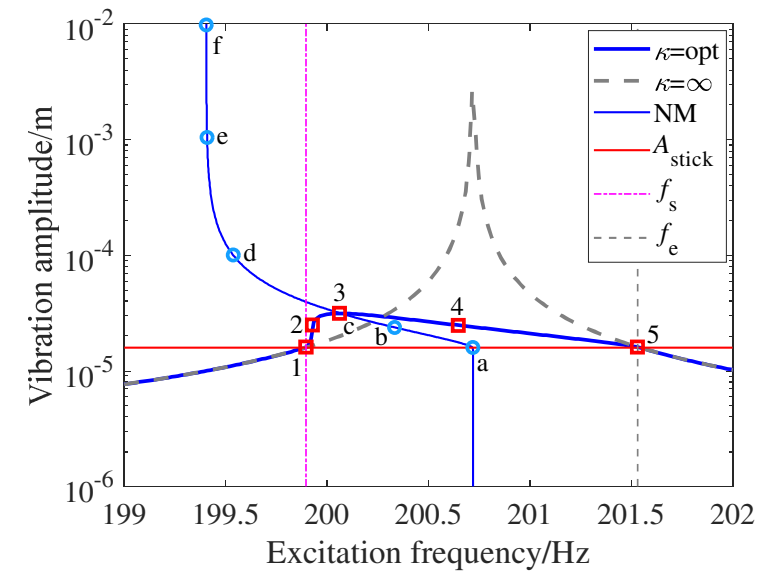

(c)

Fig. 6 Nonlinear forced responses of the flywheel (a), nonlinear modes (b), and comparison of the nonlinear forced response and the nonlinear mode (c) at the optimal value of $\kappa$. 
The time history of the friction force and the corresponding hysteresis loop at points 1-5 in Figure 6(c) are showed in Figures 7(a) and (b), respectively. The time history of the displacement of the friction contact point and slip ratio are displayed in Figures 7(c) and (d), respectively.

As shown in Figures 7(a) and (b), the horizontal and inclined sections of the friction force curves correspond to the slip and the stick states, respectively. In contrast, for the displacement curves of the friction contact point, the horizontal and inclined sections correspond to the stick and slip states, respectively (Figure 7(c)).

In Figure 7(d), the slip ratio is defined as the ratio of the time that the friction contact point is in the fully slip state to the total period of a vibration; the slip ratio can be observed to be in the range of 1045\%. At points 1 and 5, the friction contact interface is in the fully stick state, i.e., no slip occurs. Therefore, the slip ratios, areas of the hysteresis loops, and displacements of the friction contact point are all zero, and the time histories of the friction forces are harmonic.

As shown in Figure 7, as the frequency increases from point 1 to 5, the area of the hysteresis loop, displacement of the friction contact point, and slip ratio of the friction contact interface increase rapidly from zero and then decrease slowly to zero. This observation is consistent with the law of damping ratio change with modal amplitude (Figure 6(b)).

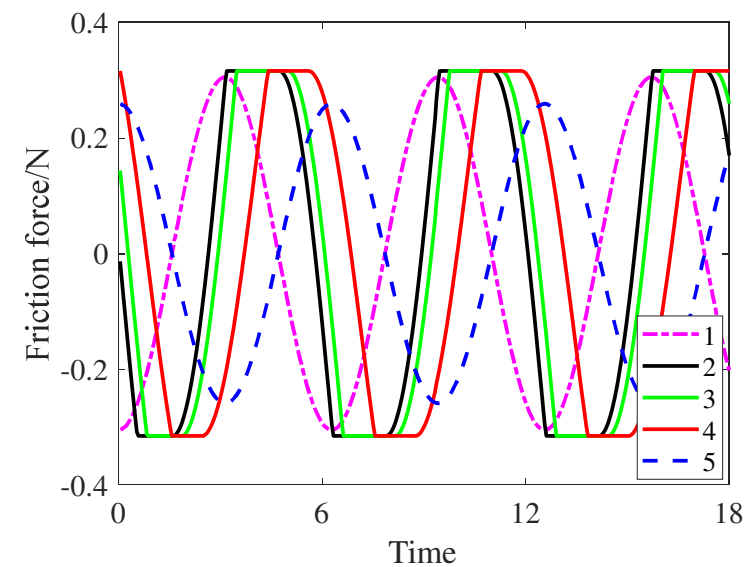

(a)

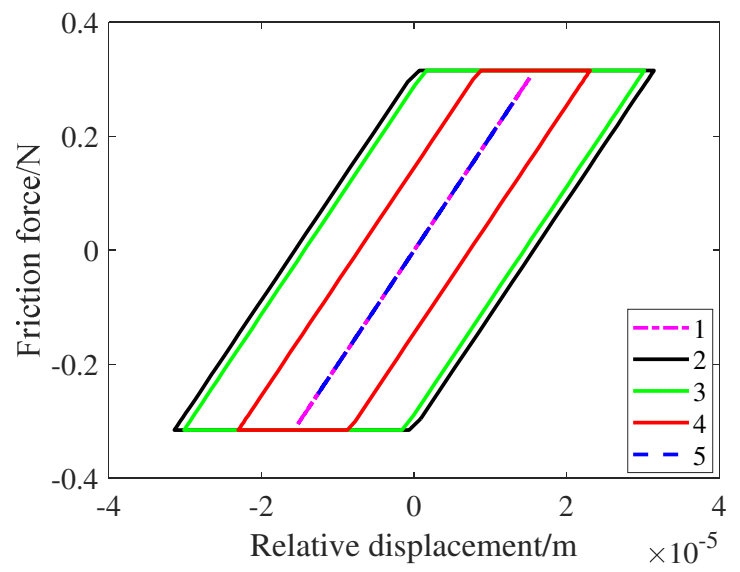

(b) 


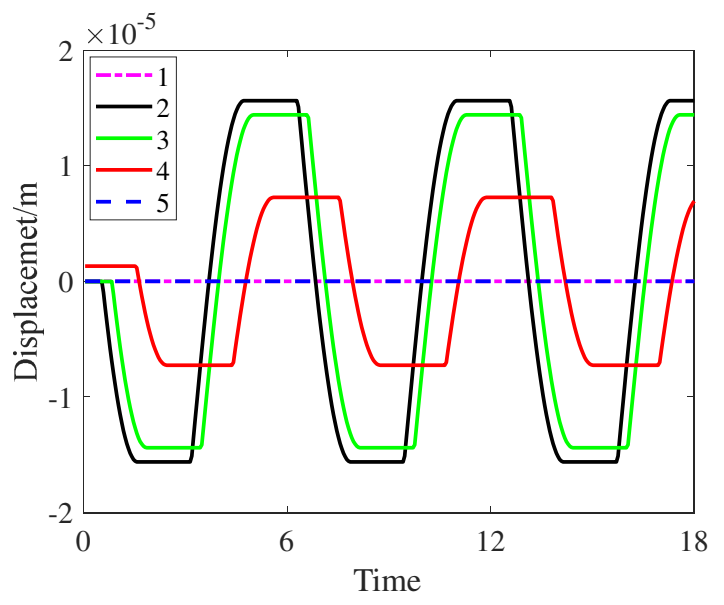

(c)

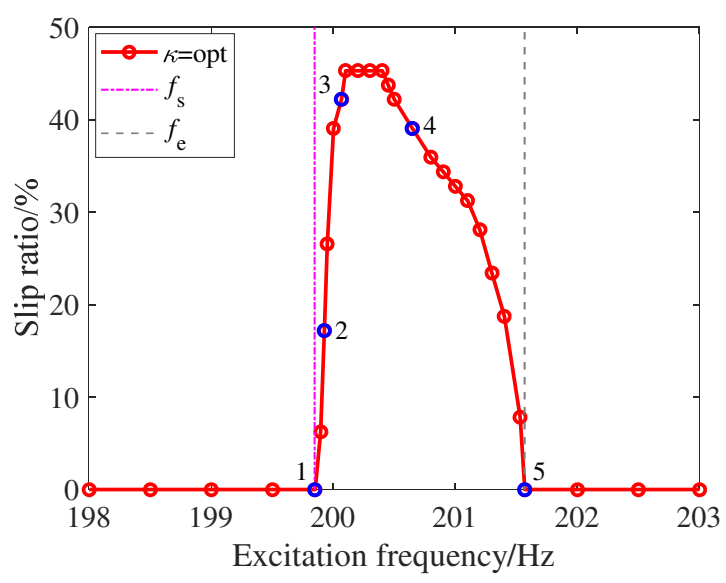

(d)

Fig. 7 Time history (a) and hysteresis loop (b) of the friction force, time history of the displacement of the friction contact point (c), and slip ratio of the friction contact interface (d) at different frequencies.

\section{Experimental validation}

Spectral testing of the flywheel was conducted with and without the friction ring damper to confirm its vibration suppression effect. Photographs of the spectral testing site are shown in Figure 8. Random excitations were produced through the vibrating table (Figure 8(a)), and acceleration data measured using the accelerometers (Figure 8(b)).

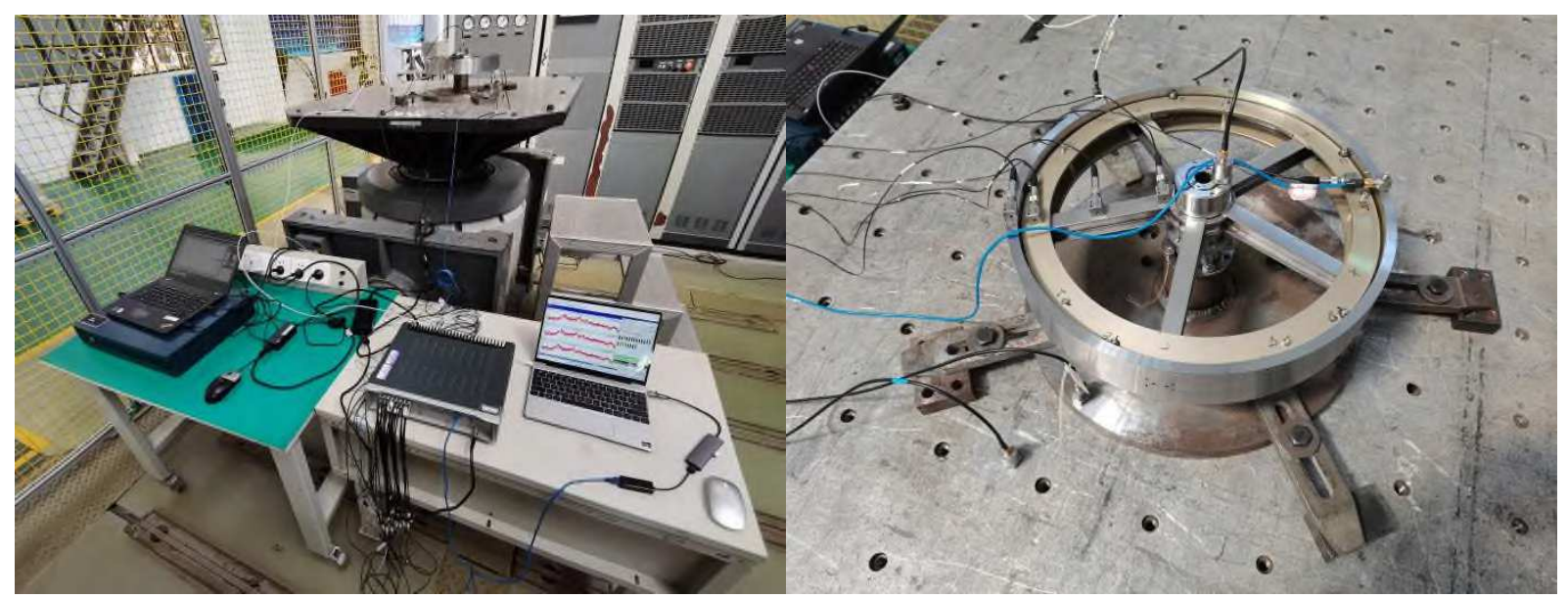

(a)

(b)

Fig. 8 Spectral testing of the flywheel (a) and layout of the accelerometers (b).

The experimental results are presented in Figure 9. Figures 9(a) and (b) show the time history of the accelerations of the rim of the flywheel and the corresponding accelerations of the vibrating table (named 
as 'base'), respectively, without and with the friction ring damper. Figure 9(c) shows the acceleration power spectral density (PSD) of the vibrating table. Figure 9(d) compares the acceleration PSD of the flywheel rim without and with the friction ring damper. According to Figure 9(d), the maximum acceleration PSD of the flywheel without the friction ring damper is $2.706 \mathrm{~g}^{2} / \mathrm{Hz}$ at the frequency 206.8 Hz, and the maximum acceleration PSD of the flywheel with the friction ring damper is $1.659 \mathrm{~g}^{2} / \mathrm{Hz}$ at the frequency $200.7 \mathrm{~Hz}$. The maximum acceleration PSD of the flywheel is reduced by $38.69 \%$ with the damper as compared to that without the damper.

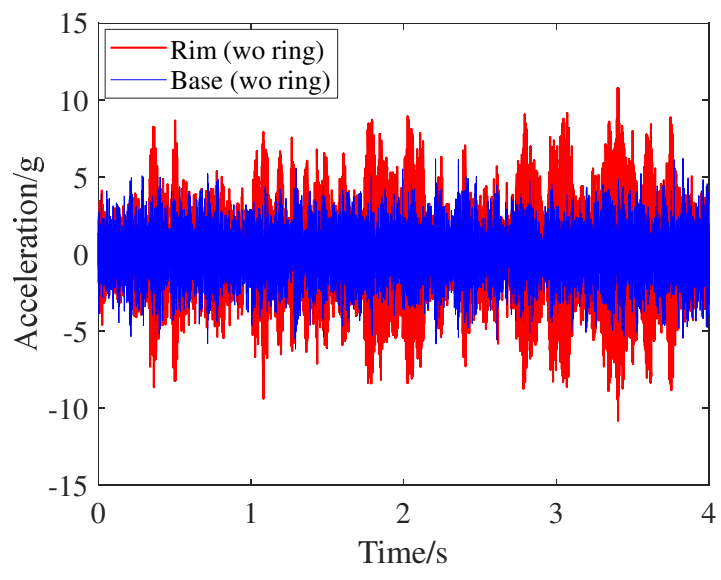

(a)

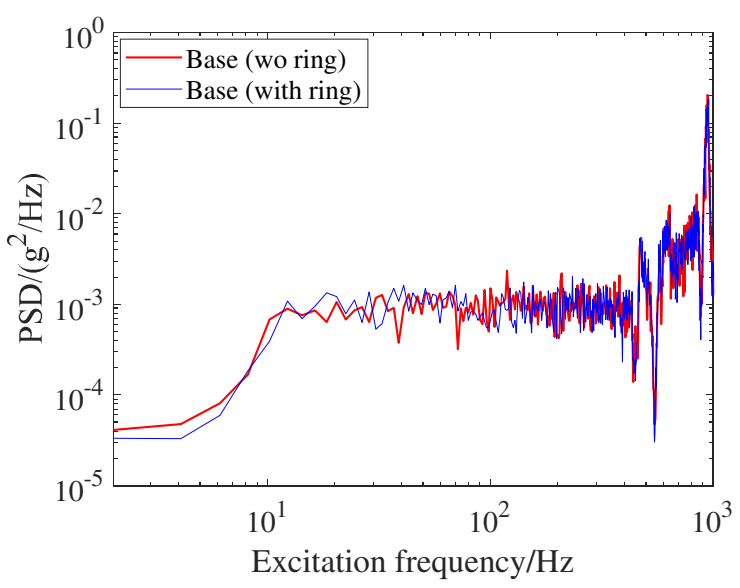

(c)

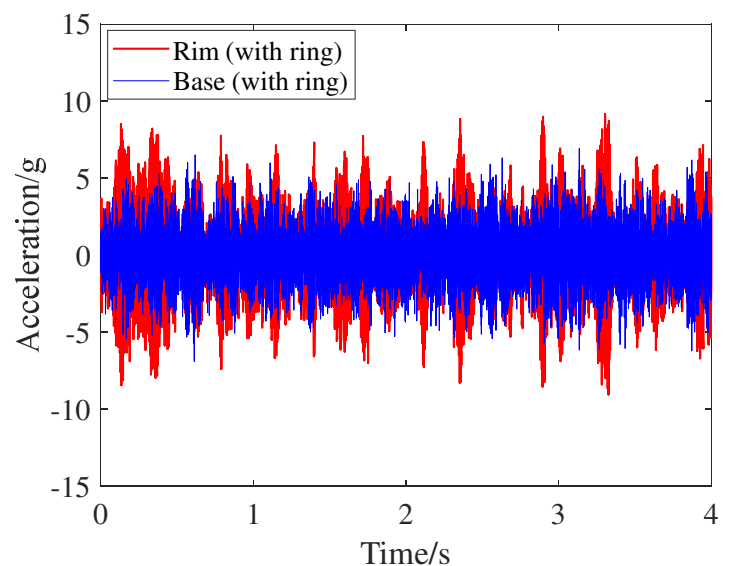

(b)

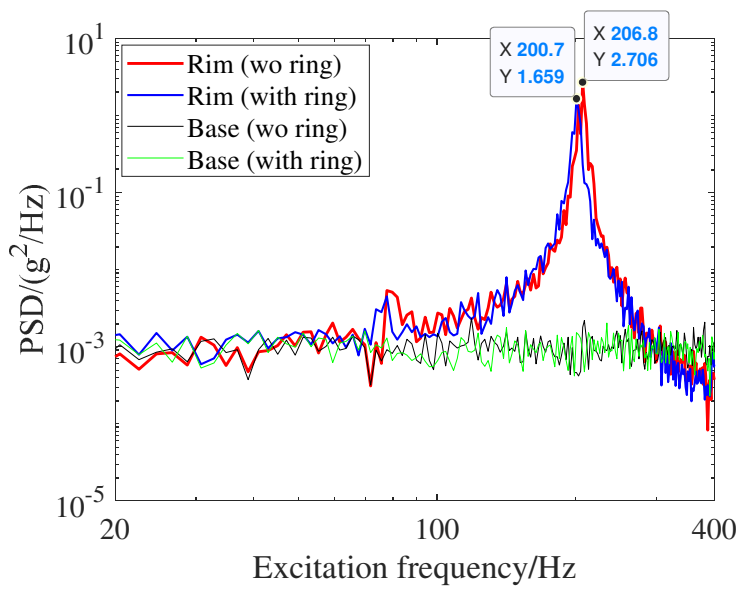

(d)

Fig. 9 Time history of the acceleration of the flywheel and the vibrating table without (a) and with (b) the friction ring damper, acceleration PSD of the vibrating table (c), and comparison of the acceleration PSD without and with the friction ring damper $(\mathrm{d})$. 
The measured acceleration of the vibrating table and acceleration PSD of the flywheel without and with the friction ring damper, shown in Figure 9, were used to verify the numerical results. In the lumpedparameter model, we removed the harmonic excitation force $F$ and replaced the fixed foundation under the bearing stiffness with the random excitation of the vibrating table shown in Figures 9(a) and (b). The fourth-order Runge-Kutta method was used to calculate the acceleration PSD of the flywheel. In this way, the vibration characteristics of the flywheel without and with the friction ring damper with actual random excitation of the vibrating table were obtained. The comparison between the numerical results and the experimental results of the acceleration PSD are shown in Figures 10(a) and (b), respectively. It can be seen that the numerical results were consistent with the experimental results.

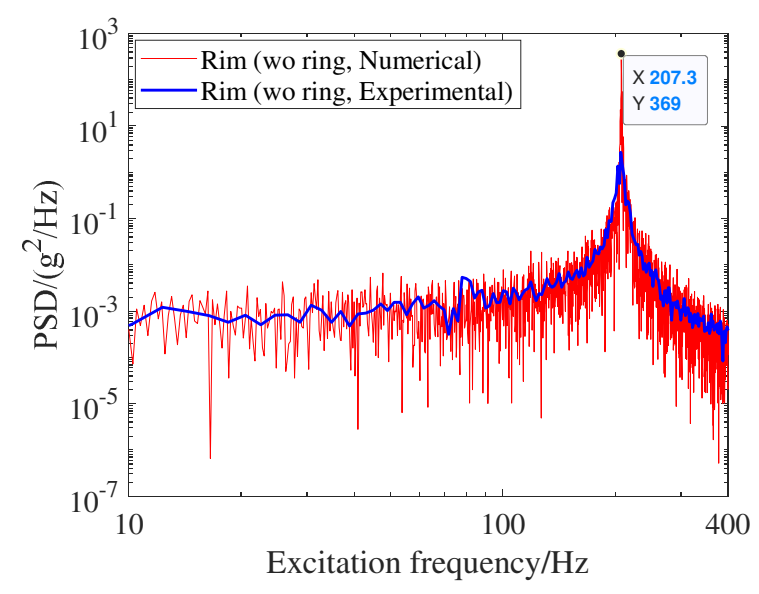

(a)

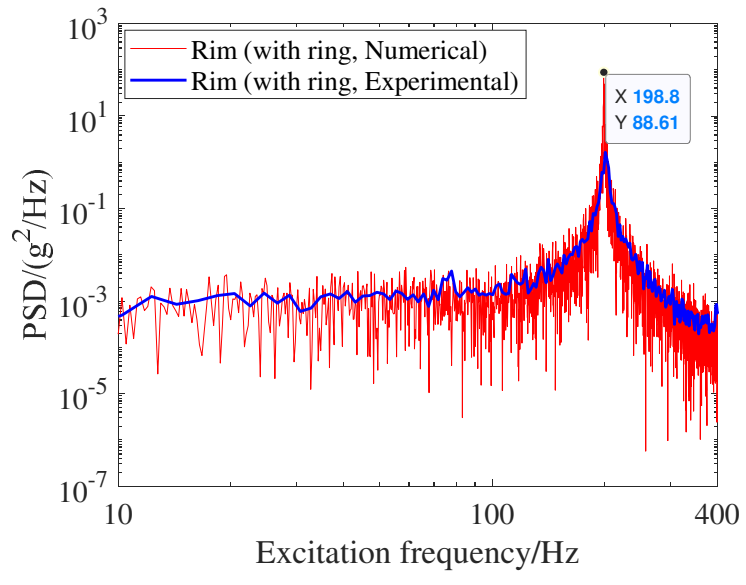

(b)

Fig. 10 Comparison of the acceleration PSD of the flywheel obtained by the Runge-Kutta method and from the spectral testing without (a) and with (b) the friction ring damper.

The friction forces under the random excitation of the vibrating table are shown in Figure 11. As shown in Figure 11(a), the friction force is no longer periodic. Figure 11(b) shows that when the spring force $F_{\mathrm{kt}}$ of the tangential stiffness spring $k_{\mathrm{t}}$ exceeds the critical friction force $\mu_{\mathrm{f}} N$, slip occurs at the friction contact interface. As shown in Figure 11(c), the friction curve is no longer a closed loop; instead, it consists of a series of combined stick-slip fold lines. The fast Fourier transform was performed on the friction force to obtain the friction force in the frequency domain, as shown in Figure 11(d), where it can 
be seen that the friction force peaks at the resonance frequency $(198.8 \mathrm{~Hz})$ of the flywheel and at its double $(397.5 \mathrm{~Hz})$ and triple resonance frequencies $(596.3 \mathrm{~Hz})$.

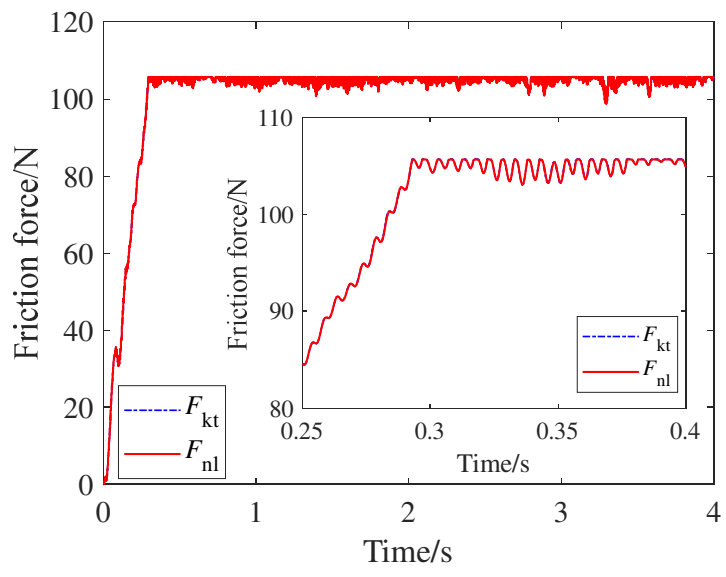

(a)

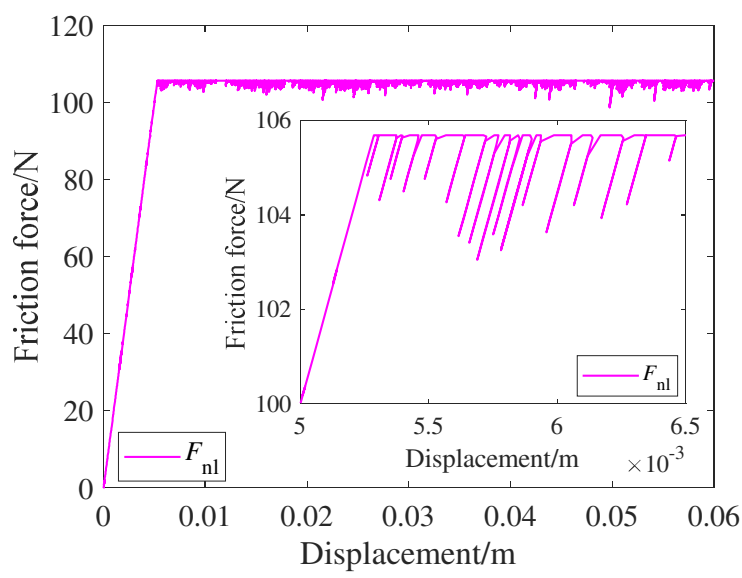

(c)

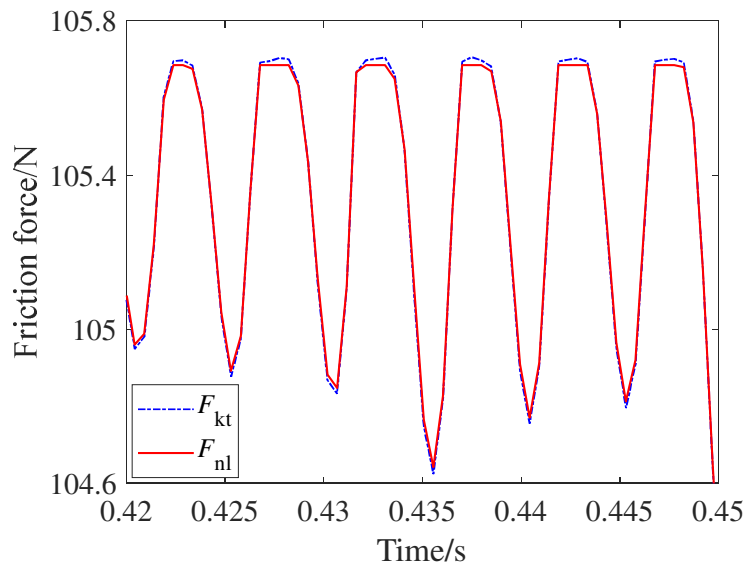

(b)

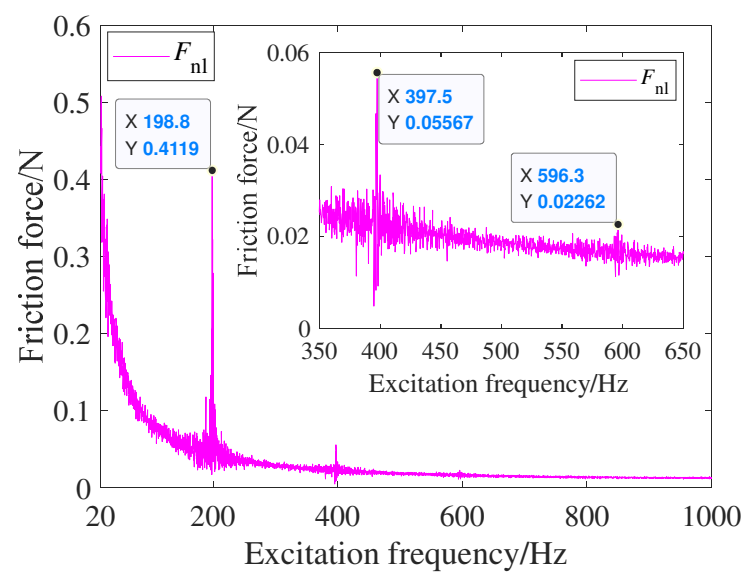

(d)

Fig. 11 Time history of the friction force (a)(b), friction force-displacement curve of the flywheel (c), and friction force in the frequency domain (d) under the random excitation.

The time history of the displacement of the friction contact point under the random excitation of the vibrating table was obtained and is shown in Figure 12(a). The displacement curve of the friction contact point shows a staircase shape, where the horizontal and inclined sections correspond to the stick and slip states of the friction contact interface, respectively. In each step of the stairs, we define the duration of the inclined section divided by the sum of the durations of the horizontal and the inclined sections as the slip ratio in a step. The slip ratios of all the steps were obtained, as shown in Figure 12(b). It can be seen 
that the slip ratios of most stair steps are in the range of $10-50 \%$, which is the same as the range of the slip ratio under the optimal value of $\kappa$ shown in Figure 7(d).

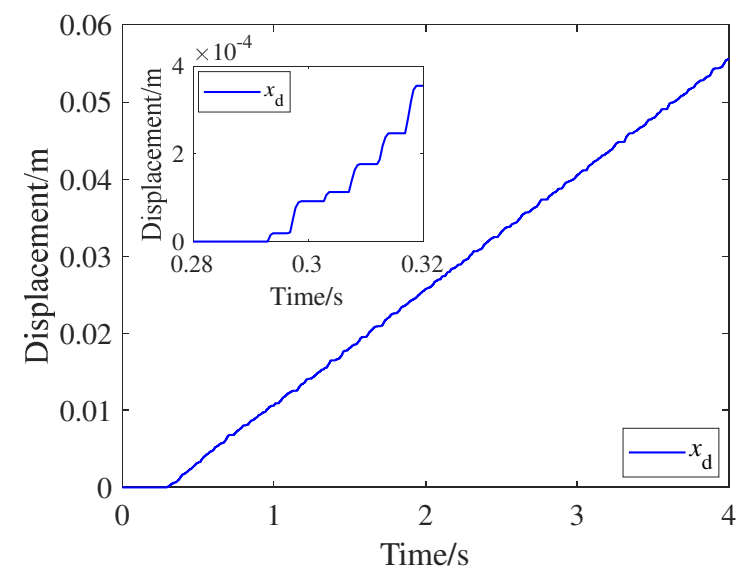

(a)

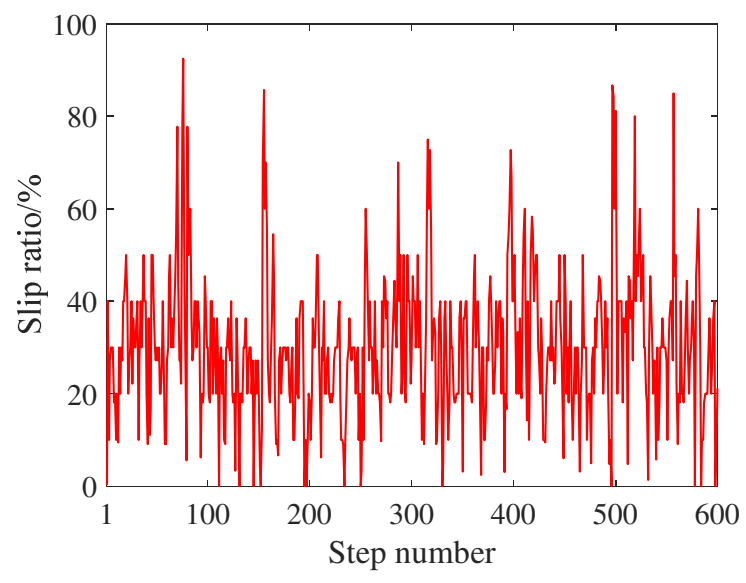

(b)

Fig. 12 Time history of the displacement of the friction contact point (a) and slip ratio of the friction contact interface (b) under random excitation.

\section{Conclusions}

A damping strategy for an industrial flywheel using a friction ring damper was investigated numerically and experimentally. The vibration reduction performance of the damper was predicted based on the established lumped-parameter model of the flywheel with friction contact interfaces. The model was numerically analyzed using the HBM combined with AFT to obtain the nonlinear forced responses under harmonic excitation, nonlinear modes of the flywheel equipped with the friction ring damper, and the behaviors of the friction contact interface. The damping performance of the friction ring damper is discussed based on numerical results. The results show that when the friction contact interface is in the fully stick state, the frequency increases monotonically as the tangential stiffness increases. A dimensionless parameter $\kappa$ is defined to evaluate the damping performance, and it is shown that an optimal value of $\kappa$ exists, which can minimize the vibration amplitude of the flywheel. In addition, all the damping ratio-frequency curves are completely coincident even with different values of $\kappa$, and the frequency at the maximum damping ratio is equal to that at the intersection of the forced response curves 
under the fully slip and the fully stick states. When the friction contact interface is in the combined stickslip state, the friction contact interface is able to provide damping. The friction interface behaviors under random excitation were also analyzed. The slip ratio is defined as the ratio of the time when the friction contact point is in the fully slip state to the total period of a vibration, which is shown to be in the range of $10-45 \%$ at the optimal value of $\kappa$. Finally, spectral testing was conducted to confirm the vibration suppression effect of the friction ring damper and experimentally verify the numerical results.

\section{Acknowledgement}

The authors acknowledge the support of the National Natural Science Foundation of China (NSFC) (Grant Nos. 51875336 and 12121002), Open Fund of State Key Laboratory of Mechanical System and Vibration (Grant No. MSVZD202001), Oceanic Interdisciplinary Program of Shanghai JiaoTong University (Grant No. SL2021MS007), and Fundamental Research Funds for the Central Universities.

\section{Declaration of conflicting interests}

The authors declare no conflict of interests in preparing this article.

\section{References}

[1] Huang, X., Su, Z., Wang, S., Wei, X., Wang, Y., Hua, H.: High-frequency disturbance force suppression mechanism of a flywheel equipped with a flexible dynamic vibration absorber. J. Vib. Control 26(23-24), 2113-2124 (2020)

[2] He, X., Huang, X., Hua, H.: Performance of a friction ring DVA for vibration control of a flywheel. InterNoise2021, 2449-2457 (2021)

[3] Huang, X., He, X., Su, Z., Hua, H., Zhang, Z.: Micro-vibration control of flywheels with friction rings based on DVA with dry friction interfaces. ICSV27 (2021)

[4] Griffin, J.H.: A review of friction damping of turbine blade vibration. Int. J. Turbo. Jet. Eng. 7(34), 297-307 (1990) 
[5] Laxalde, D., Sinou, J.J., Thouverez, F., Lombard, J.P.: Modeling and analysis of friction rim dampers for blisks. Proceedings of IDETC/CIE 2005, 1013-1021 (2005)

[6] Krack, M., Gross, J.: Harmonic balance for nonlinear vibration problems. Springer Nature, Switzerland (2019)

[7] Laxalde, D., Thouverez, F., Sinou, J.J., Lombard, J.P.: Qualitative analysis of forced response of blisks with friction ring dampers. Eur. J. Mech. A/Solid 26(4), 676-687 (2007)

[8] Petrov, E.P., Ewins, D.J.: Analytical formulation of friction interface elements for analysis of nonlinear multiharmonic vibrations of bladed disks. J. Turbomach. 125(2), 364-371 (2003)

[9] Nacivet, S., Pierre, C., Thouverez, F., Jezequel, L.: A dynamic Lagrangian frequency-time method for the vibration of dry-friction-damped systems. J. Sound Vib. 265(1), 201-219 (2003)

[10] Krack, M., Salles, L., Thouverez, F.: Vibration prediction of bladed disks coupled by friction joints. Arch. Computat. Methods Eng. 24, 589-636 (2017)

[11] Sun, Y., Yuan, J., Pesaresi L., Salles, L.: Nonlinear vibrational analysis for integrally bladed disk using frictional ring damper. J. Phys.: Conf. Ser. 1106, 012026 (2018)

[12] Laxalde, D., Salles, L., Blanc, L., Thouverez, F.: Non-linear modal analysis for bladed disks with friction contact interfaces. Proceedings of ASME Turbo Expo 2008: power for land, sea and air, 457-467 (2008)

[13] Sun, Y., Yuan, J., Denimal, E., Salles, L.: Nonlinear modal analysis of frictional ring damper for compressor blisk. J. Eng. Gas Turbines Power 143(3), 031008 (2021)

[14] Kerschen, G.: Modal analysis of nonlinear mechanical systems. Springer, New York (2014)

[15] Laxalde, D., Thouverez, F.: Complex non-linear modal analysis for mechanical systems: Application to turbomachinery bladings with friction interfaces. J. Sound Vib. 322(4-5), 1009-1025 (2009)

[16] Cameron, T.M., Griffin, J.H.: An alternating frequency/time domain method for calculating the steady-state response of nonlinear dynamic systems. J. Appl. Mech. 56(1), 149-154 (1989) 
[17] Seydel, R.: Practical bifurcation and stability analysis. Springer, New York (2009)

[18] Liu, J., Li, L., Fan, Y.: A comparison between the friction and piezoelectric synchronized switch dampers for blisks. J. Intel. Mat. Syst. Str. 29(12), 2693-2705 (2018)

[19] Liu, J., Li, L., Huang, X, Jezequel, L.: Dynamic characteristics of the blisk with synchronized switch damping based on negative capacitor. Mech. Syst. Signal Pr. 95, 425-445 (2017)

[20] Krack, M.: Nonlinear modal analysis of nonconservative systems: Extension of the periodic motion concept. Comput. Struct. 154, 59-71 (2015)

[21] Andersson, S., Söderberg, A., Björklund, S.: Friction models for sliding dry, boundary and mixed lubricated contacts. Tribol. Int. 40(4), 580-587 (2007)

\section{Statements \& Declarations}

The authors acknowledge the support of the National Natural Science Foundation of China (NSFC) (Grant Nos. 51875336 and 12121002), Open Fund of State Key Laboratory of Mechanical System and Vibration (Grant No. MSVZD202001), Oceanic Interdisciplinary Program of Shanghai JiaoTong University (Grant No. SL2021MS007), and Fundamental Research Funds for the Central Universities.

The authors declare no conflict of interests in preparing this article.

All authors contributed to the study conception and design. Material preparation, data collection and analysis were performed by Xiaodong He, Xiuchang Huang and Zhiwei Zheng. The first draft of the manuscript was written by Xiaodong $\mathrm{He}$ and all authors commented on previous versions of the manuscript. All authors read and approved the final manuscript.

The datasets generated during the current study are available from the corresponding author on reasonable request. 
Table 1 Parameter values of the lumped-parameter model.

\begin{tabular}{|c|c|c|c|c|}
\hline$m_{\mathrm{f}}$ & $k_{\mathrm{f}}$ & $k_{\mathrm{f} 0}$ & $m_{\mathrm{b}}$ & $k_{\mathrm{b}}$ \\
\hline $0.9105 \mathrm{~kg}$ & $2.824 \times 10^{6} \mathrm{~N} / \mathrm{m}$ & $1 \times 10^{7} \mathrm{~N} / \mathrm{m}$ & $1.0710 \mathrm{~kg}$ & $1.6150 \times 10^{7} \mathrm{~N} / \mathrm{m}$ \\
\hline$m_{\mathrm{r}}$ & $k_{\mathrm{g}}$ & $k_{\mathrm{r} 0}$ & $c_{\mathrm{r}}$ & - \\
\hline $0.0990 \mathrm{~kg}$ & $1 \times 10^{10} \mathrm{~N} / \mathrm{m}$ & $1 \times 10^{6} \mathrm{~N} / \mathrm{m}$ & 0 & - \\
\hline
\end{tabular}




\section{Figure Captions}

Fig. 1 Umbrella-shaped flapping mode of the flywheel.

Fig. 2 Flywheel equipped with the friction ring damper (a) and the 11-DOF lumped-parameter model (b).

Fig. 3 Effect of $k_{\mathrm{t}}$ on the frequency of the flywheel.

Fig. 4 Effect of $\kappa$ on the vibration amplitude of the flywheel.

Fig. 5 Frequency-modal amplitude diagram (a), damping ratio-modal amplitude diagram (b), damping ratiofrequency diagram (c), and intersection of the forced response curves for $\kappa=0$ and $\kappa=\infty$ (d).

Fig. 6 Nonlinear forced responses of the flywheel (a), nonlinear modes (b), and comparison of the nonlinear forced response and the nonlinear mode (c) at the optimal value of $\kappa$.

Fig. 7 Time history (a) and hysteresis loop (b) of the friction force, time history of the displacement of the friction contact point (c), and slip ratio of the friction contact interface (d) at different frequencies.

Fig. 8 Spectral testing of the flywheel (a) and layout of the accelerometers (b).

Fig. 9 Time history of the acceleration of the flywheel and the vibrating table without (a) and with (b) the friction ring damper, acceleration PSD of the vibrating table (c), and comparison of the acceleration PSD without and with the friction ring damper $(d)$.

Fig. 10 Comparison of the acceleration PSD of the flywheel obtained by the Runge-Kutta method and from the spectral testing without (a) and with (b) the friction ring damper.

Fig. 11 Time history of the friction force (a)(b), friction force-displacement curve of the flywheel (c), and friction force in the frequency domain (d) under the random excitation.

Fig. 12 Time history of the displacement of the friction contact point (a) and slip ratio of the friction contact interface (b) under random excitation. 

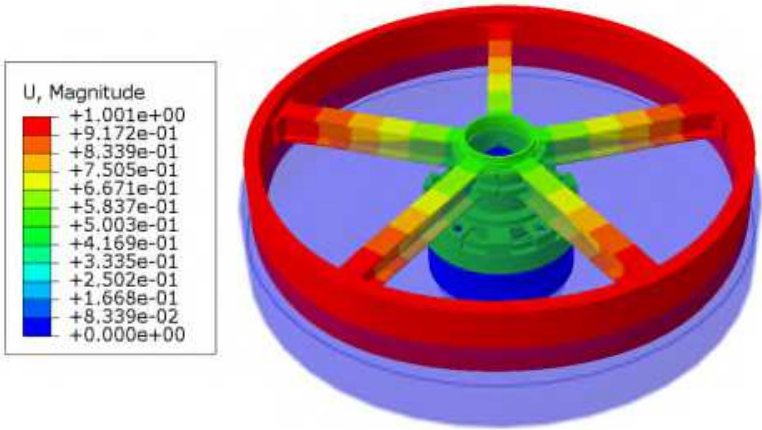

Fig. 1 Umbrella-shaped flapping mode of the flywheel. 


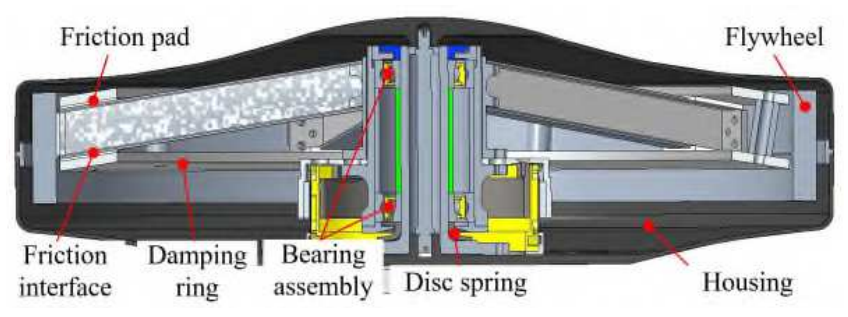

(a)

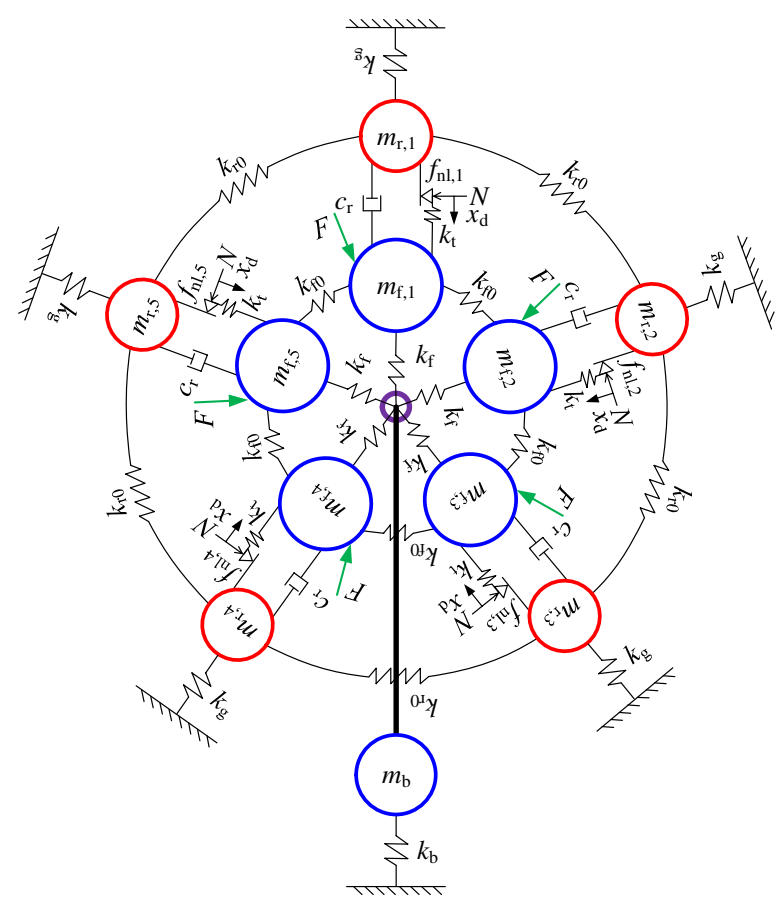

(b)

Fig. 2 Flywheel equipped with the friction ring damper (a) and the 11-DOF lumped-parameter model (b). 


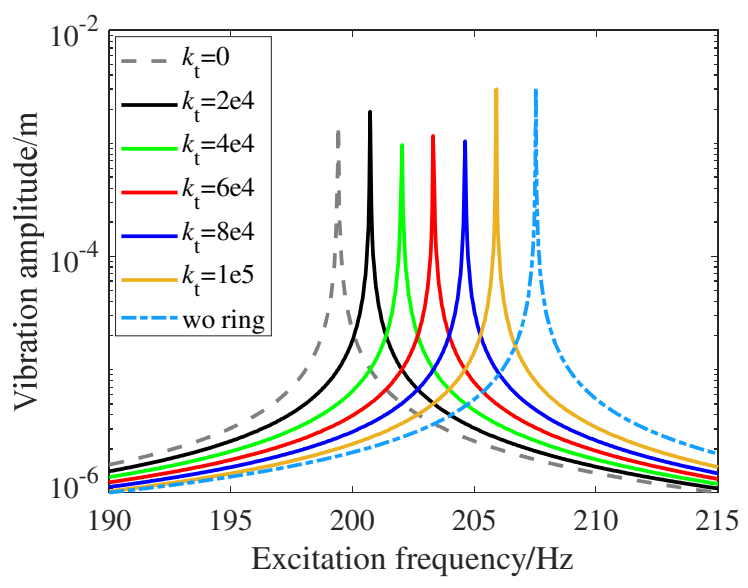

Fig. 3 Effect of $k_{\mathrm{t}}$ on the frequency of the flywheel. 


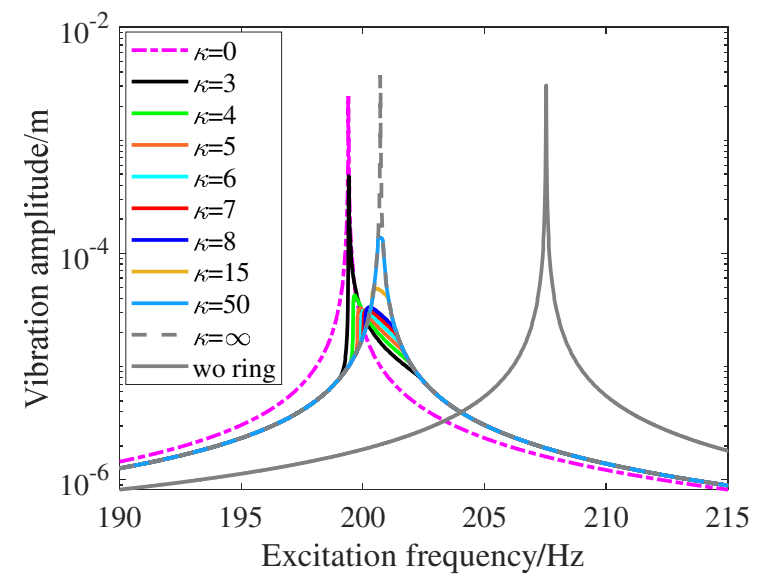

(a)

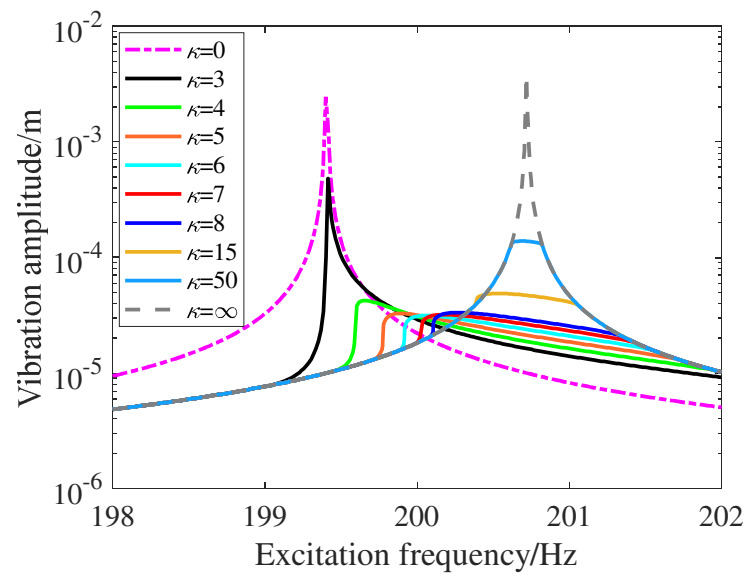

(b)

Fig. 4 Effect of $\kappa$ on the vibration amplitude of the flywheel. 


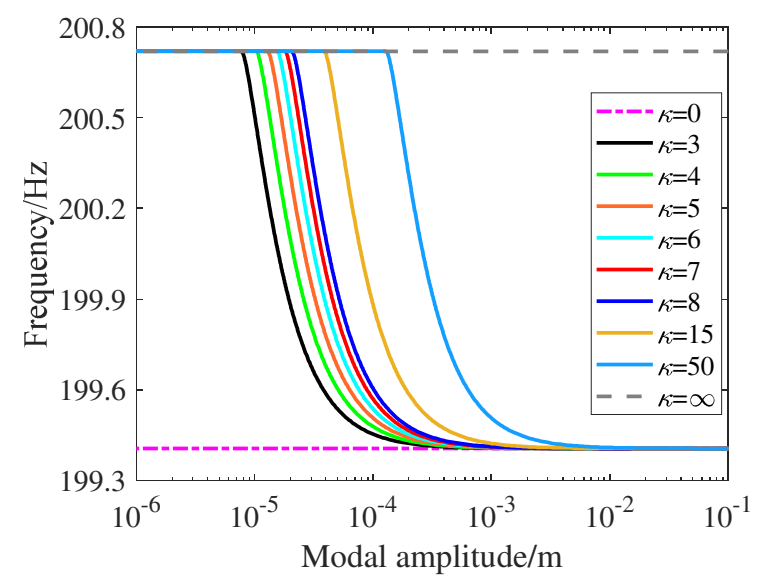

(a)

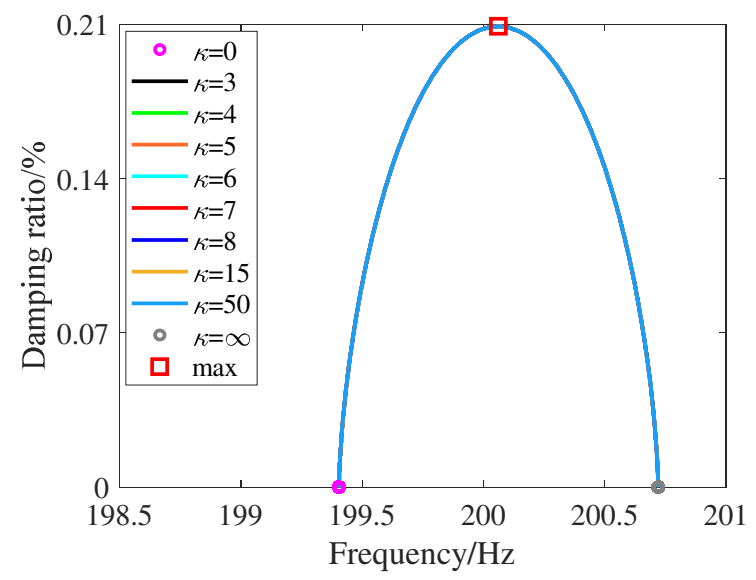

(c)

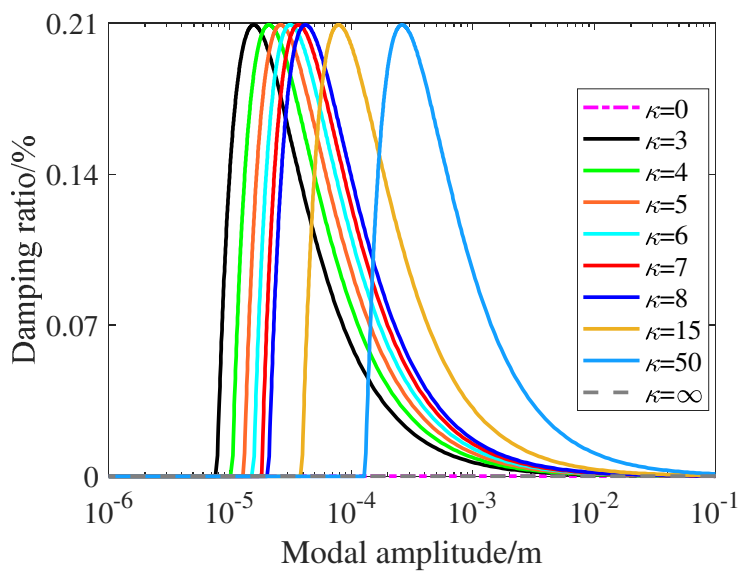

(b)

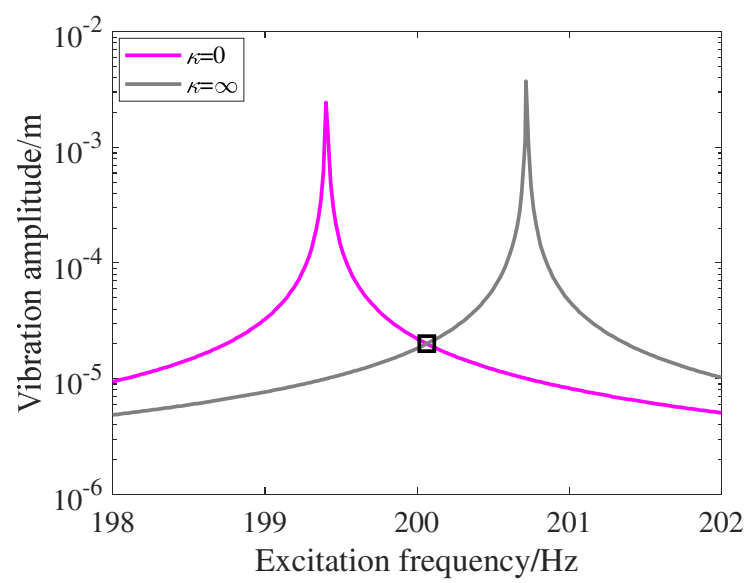

(d)

Fig. 5 Frequency-modal amplitude diagram (a), damping ratio-modal amplitude diagram (b), damping ratiofrequency diagram (c), and intersection of the forced response curves for $\kappa=0$ and $\kappa=\infty$ (d). 


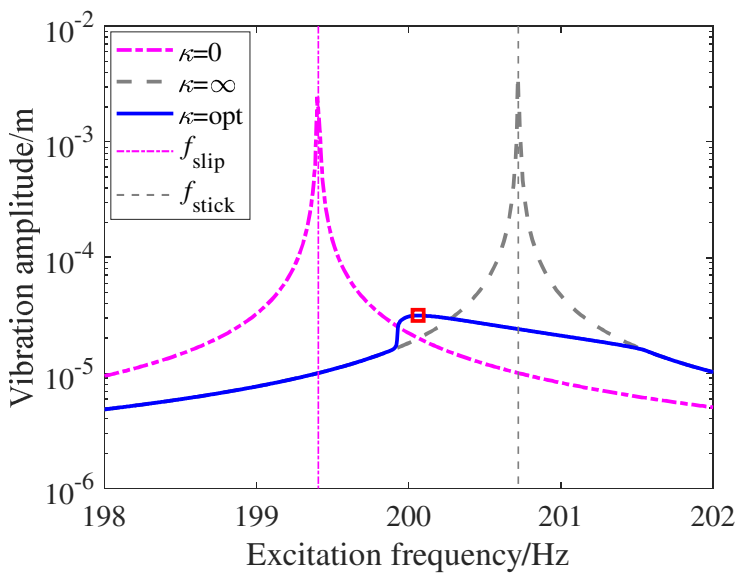

(a)
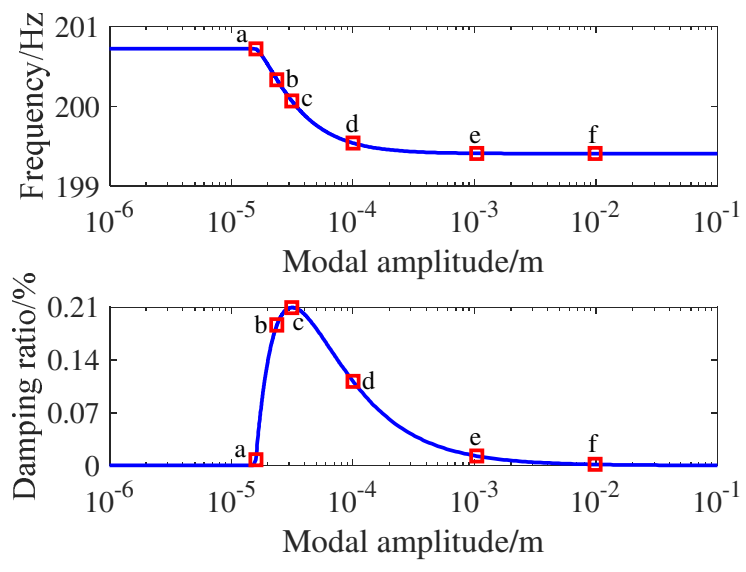

(b)

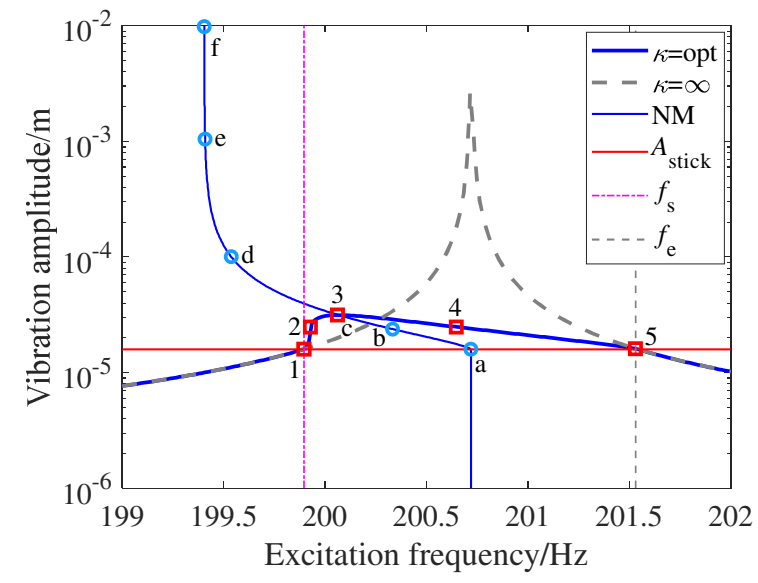

(c)

Fig. 6 Nonlinear forced responses of the flywheel (a), nonlinear modes (b), and comparison of the nonlinear forced response and the nonlinear mode (c) at the optimal value of $\kappa$. 


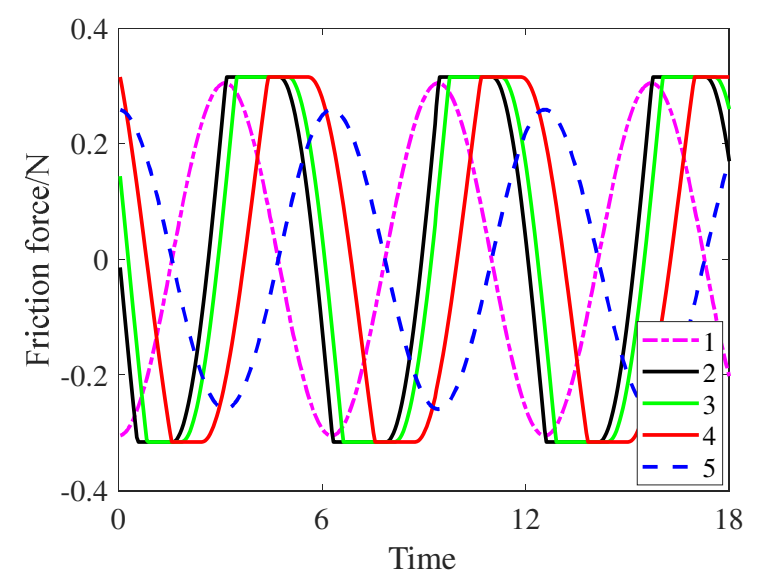

(a)

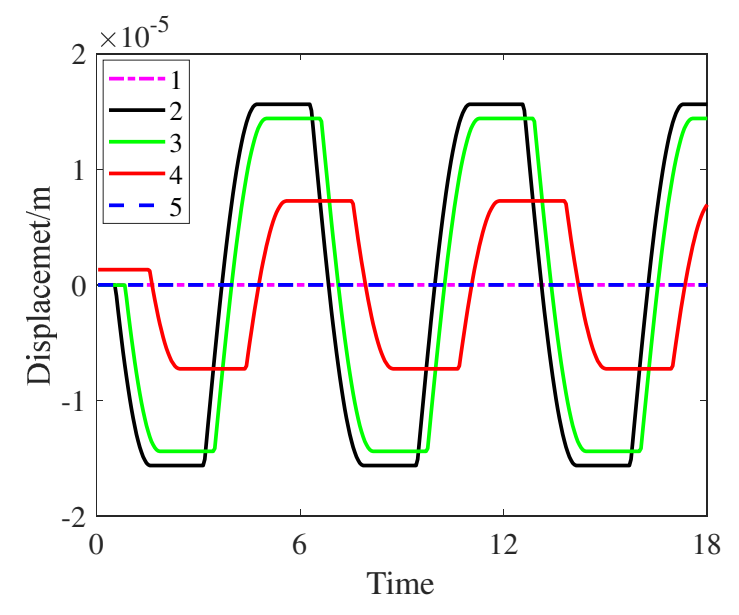

(c)

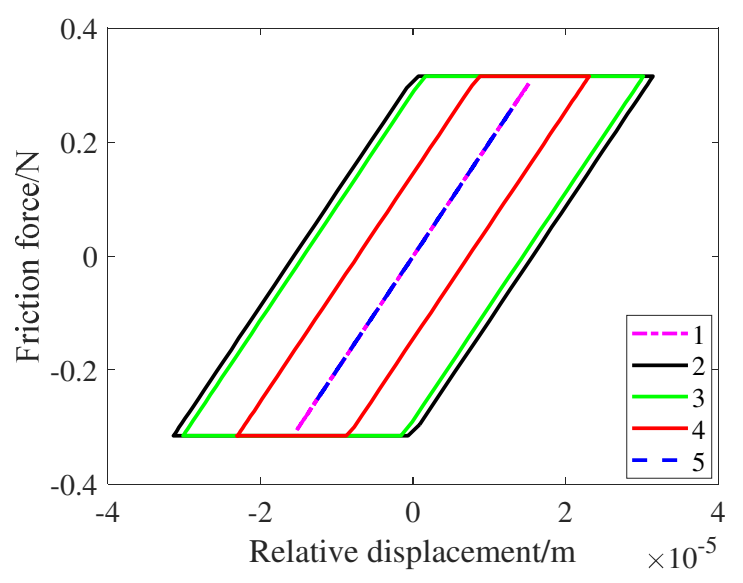

(b)

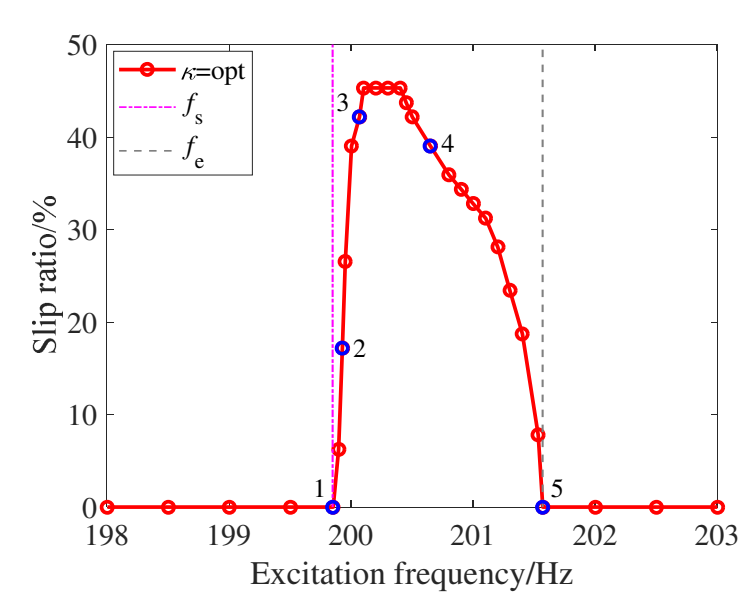

(d)

Fig. 7 Time history (a) and hysteresis loop (b) of the friction force, time history of the displacement of the friction contact point (c), and slip ratio of the friction contact interface (d) at different frequencies. 


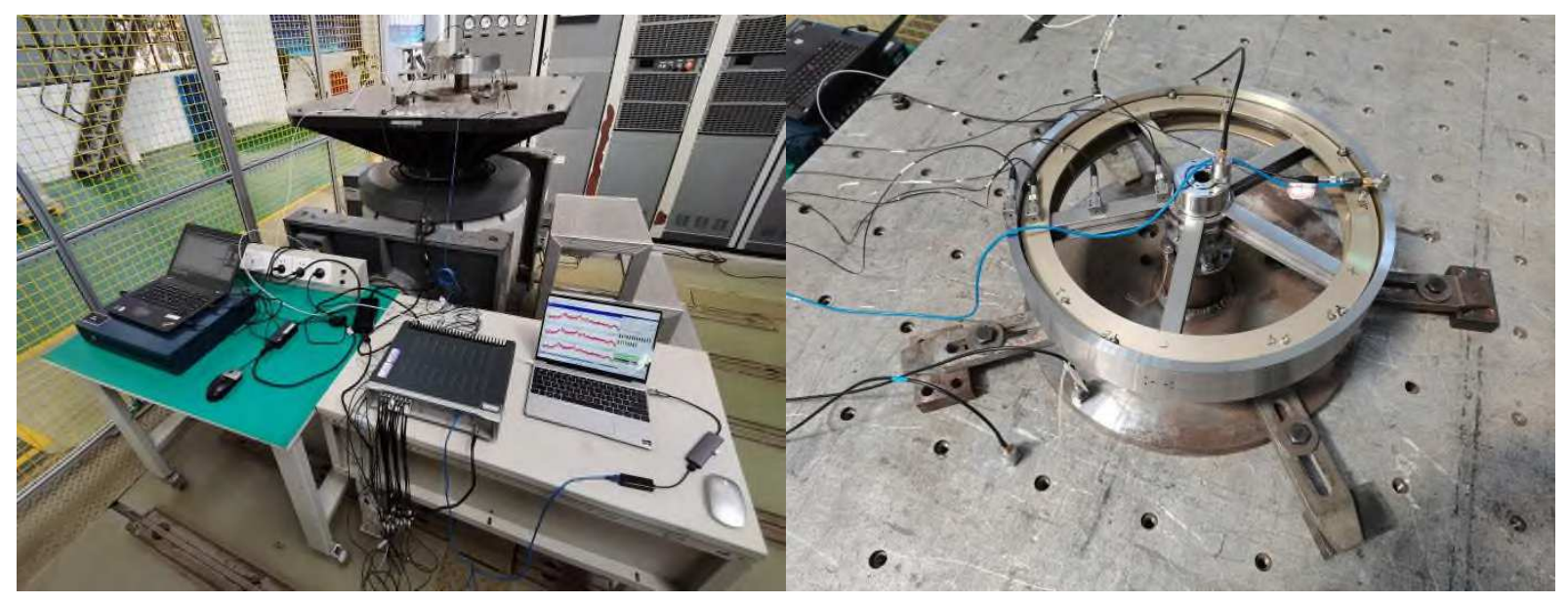

(a)

(b)

Fig. 8 Spectral testing of the flywheel (a) and layout of the accelerometers (b). 


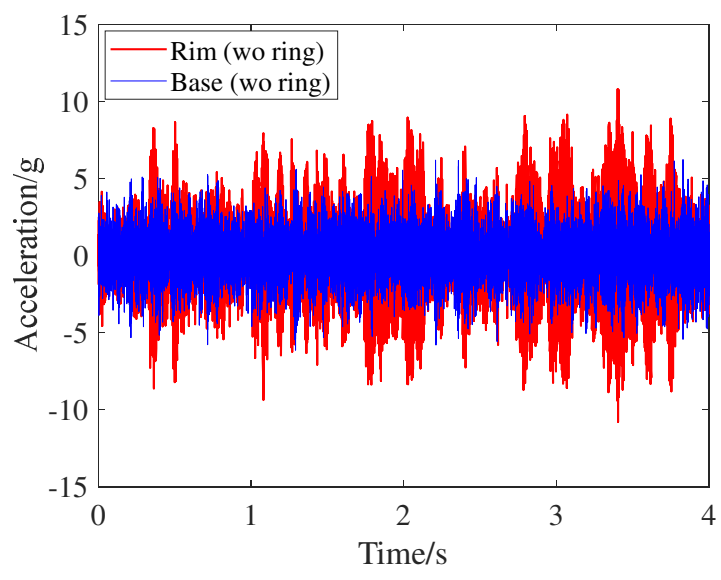

(a)

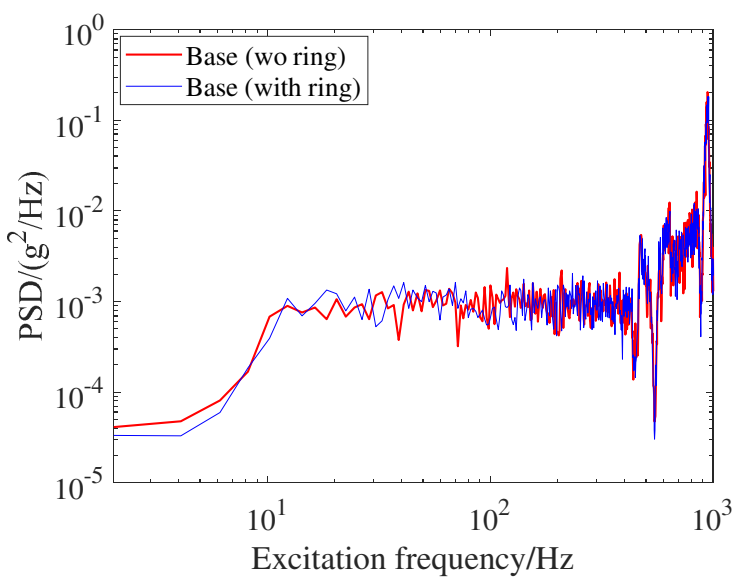

(c)

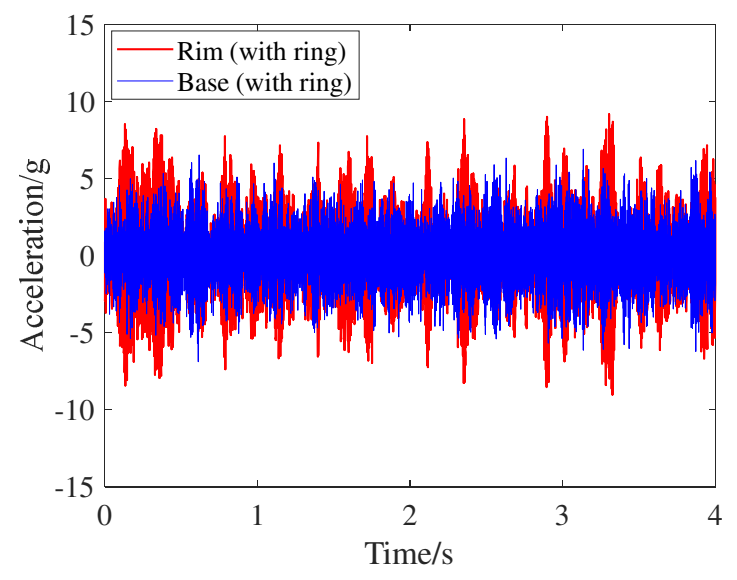

(b)

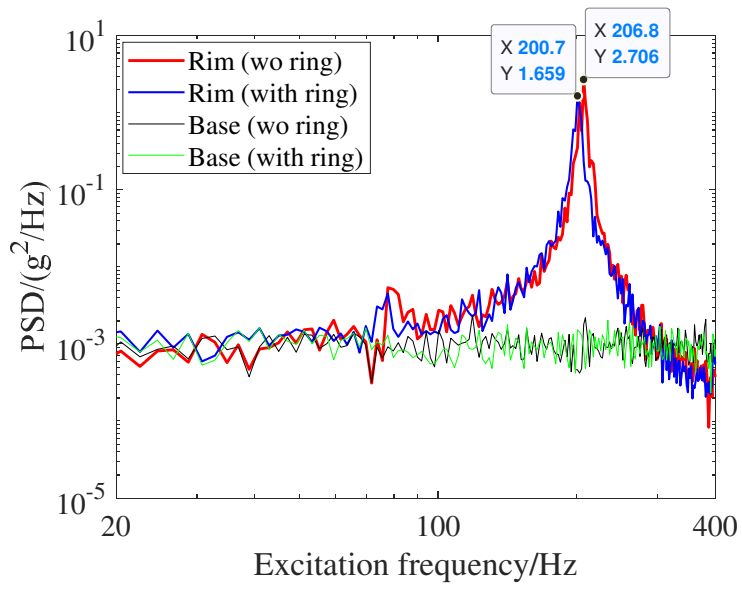

(d)

Fig. 9 Time history of the acceleration of the flywheel and the vibrating table without (a) and with (b) the friction ring damper, acceleration PSD of the vibrating table (c), and comparison of the acceleration PSD without and with the friction ring damper (d). 


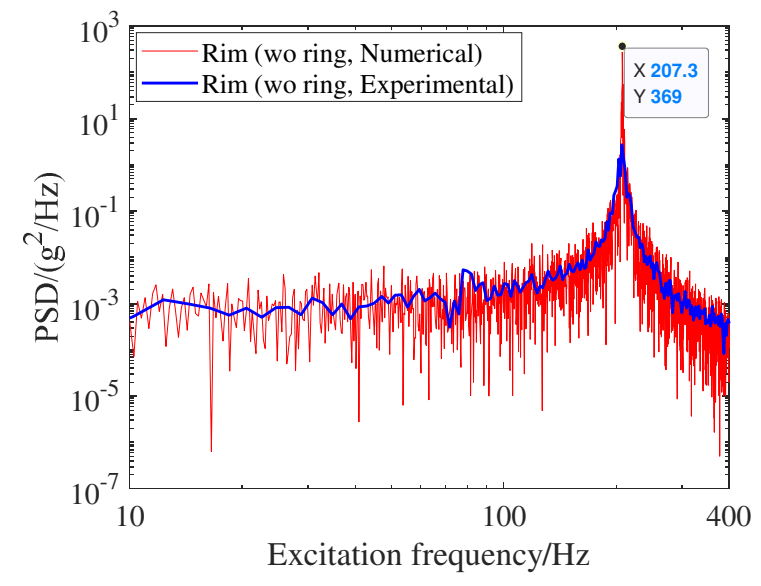

(a)

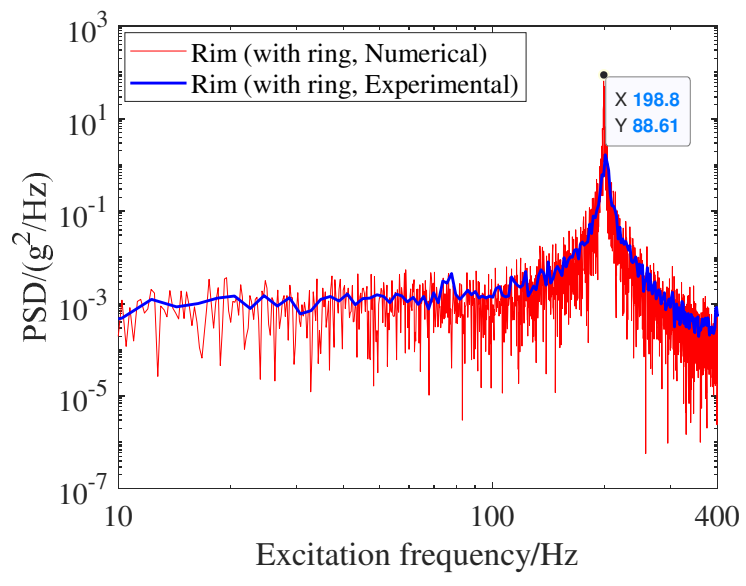

(b)

Fig. 10 Comparison of the acceleration PSD of the flywheel obtained by the Runge-Kutta method and from the spectral testing without (a) and with (b) the friction ring damper. 


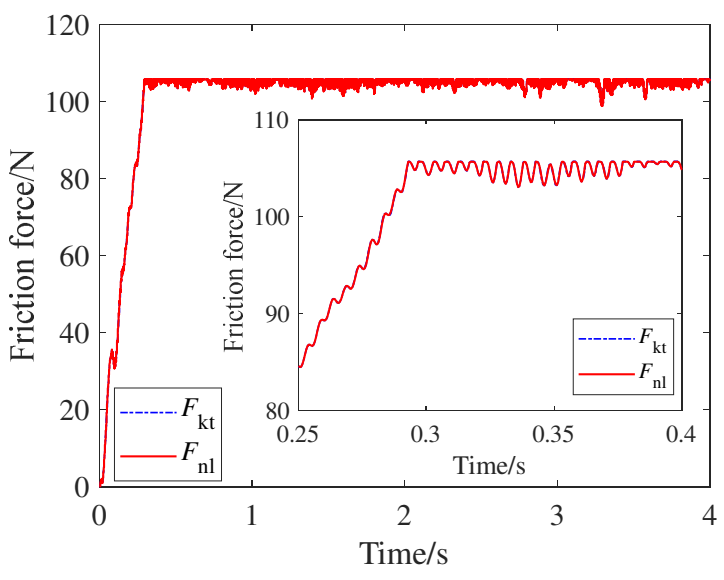

(a)

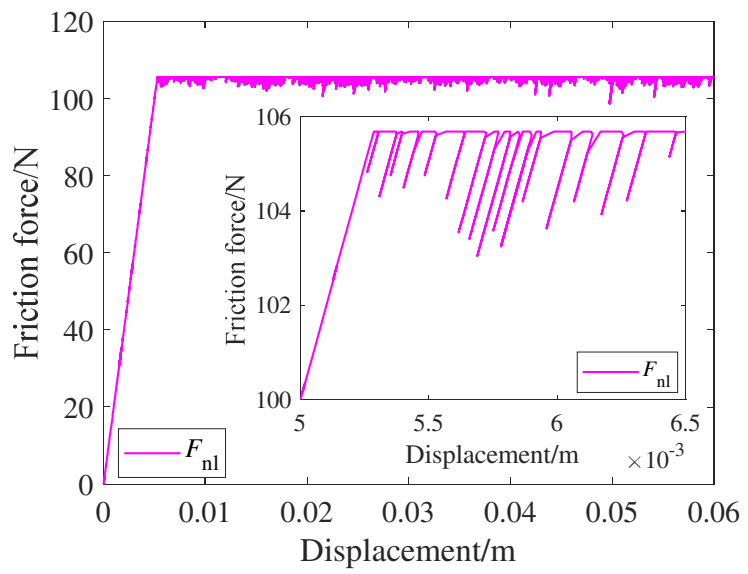

(c)

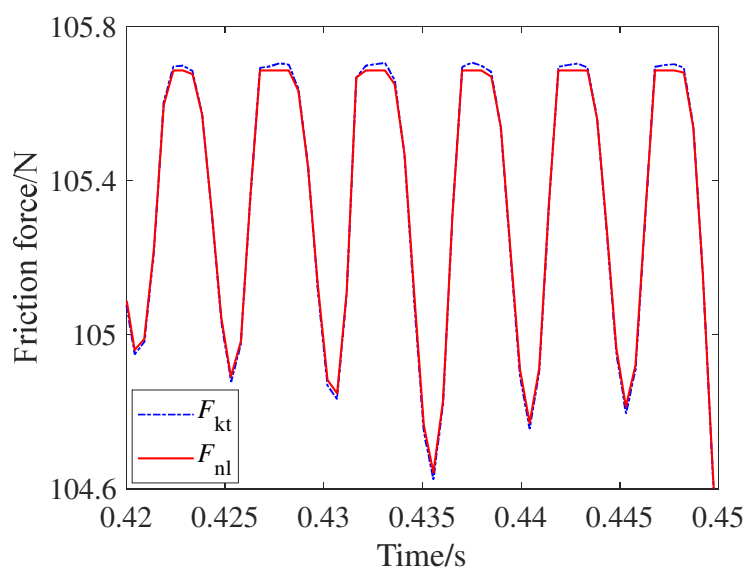

(b)

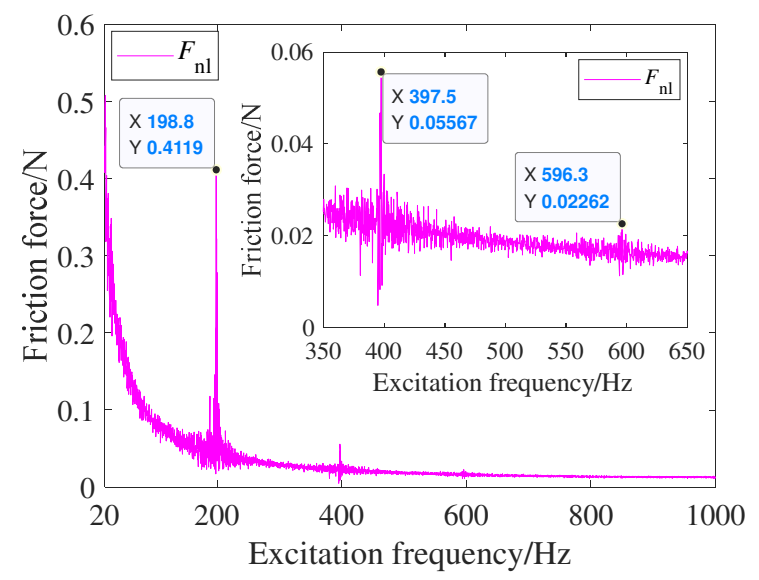

(d)

Fig. 11 Time history of the friction force (a)(b), friction force-displacement curve of the flywheel (c), and friction force in the frequency domain (d) under the random excitation. 


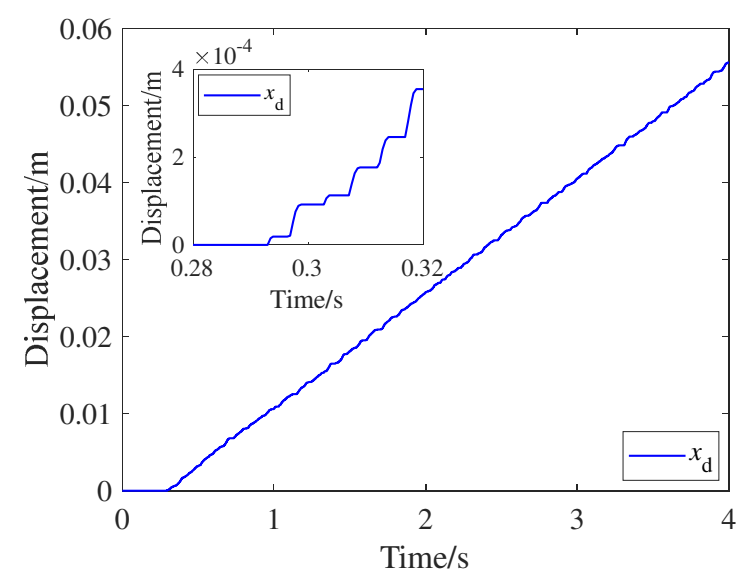

(a)

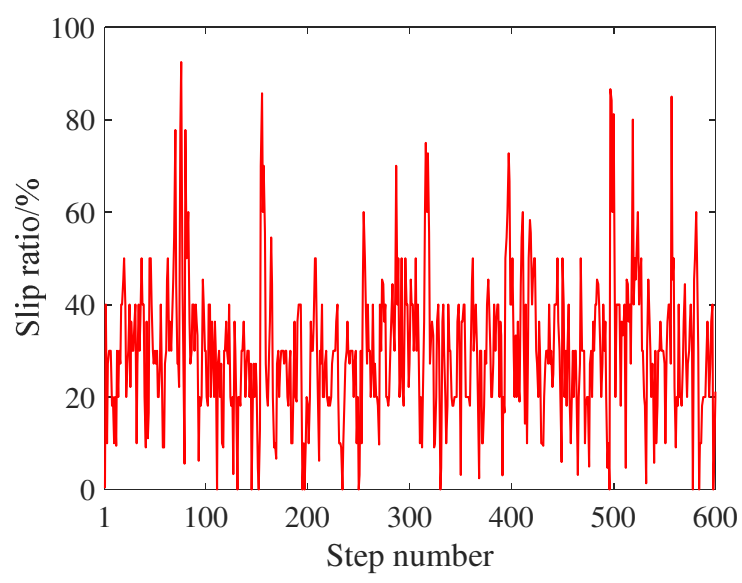

(b)

Fig. 12 Time history of the displacement of the friction contact point (a) and slip ratio of the friction contact interface (b) under random excitation. 